CERN-PH-TH/2005-152

\title{
Magnetized CMB anisotropies
}

\author{
Massimo Giovannini ${ }^{1}$ \\ Centro "Enrico Fermi", Compendio del Viminale, Via Panisperna 89/A, 00184 Rome, Italy \\ Department of Physics, Theory Division, CERN, 1211 Geneva 23, Switzerland
}

\begin{abstract}
Possible effects of large-scale magnetic fields on the Cosmic Microwave Background (CMB) are reviewed. Depending on the specific branch of the spectrum of plasma excitations, magnetic fields are treated either within a two-fluid plasma description or within an effective (one-fluid) approach. The uniform field approximation is contrasted with the fully inhomogeneous field approximation. It is argued that the interplay between CMB physics and large-scale magnetic fields will represent a rather interesting cross-disciplinary arena along the next few years.
\end{abstract}

\footnotetext{
${ }^{1}$ e-mail address: massimo.giovannini@cern.ch
} 


\section{Why CMB anisotropies could be magnetized?}

Simplified magneto-hydrodynamical estimates imply that the magnetic diffusivity length scale $^{2}$ in the interstellar medium is of the order of the astronomical unit. On the other hand, magnetic fields are present over much larger length-scales so that there seems to be rather compelling evidence that galaxies, clusters and possibly super-clusters are all magnetized. The question that arises naturally in this context concerns the possible effects of large-scale magnetic fields on the Cosmic Microwave Background (CMB). In the last fifty years various cosmological mechanisms for the origin of large scale magnetic fields have been proposed. While different mechanisms rely on diverse physical assumptions some general features can be identified:

- the majority of the cosmological mechanisms imply the existence of large-scale magnetic fields after equality (but before decoupling);

- the magnetic field present after equality is, according to the mentioned mechanisms, fully inhomogeneous;

- the typical amplitudes and length-scales of the magnetic field are characteristic of the given model.

The first possibility we can think of implies that magnetic fields are produced, at a given epoch in the life of the Universe, inside the Hubble radius, for instance by a phase transition or by any other phenomenon able to generate a charge separation and, ultimately, an electric current. In this context, the correlation scale of the field is much smaller that the typical scale of the gravitational collapse of the proto-galaxy which is of the order of the Mpc. In fact, if the Universe is decelerating and if the correlation scale evolves as the scale factor, the Hubble radius grows much faster than the correlation scale. Of course, one might invoke the possibility that the correlation scale of the magnetic field evolves more rapidly than the scale factor. A well founded physical rationale for this occurrence is what is normally called inverse cascade, i.e. the possibility that magnetic (as well as kinetic) energy density is transferred from small to large scales. This implies, in real space, that (highly energetic) small scale magnetic domains may coalesce to form magnetic domains of smaller energy but over larger scales. In the best of all possible situations, i.e. when inverse cascade is very effective, it seems rather hard to justify a growth of the correlation scale that would eventually end up into a Mpc scale at the onset of gravitational collapse. In Fig. 11 we report a schematic illustration of the evolution of the Hubble radius $R_{H}$ and of

\footnotetext{
${ }^{2}$ The magnetic diffusivity length is the typical scale below which magnetic fields are diffused because of the finite value of the conductivity of the (interstellar) medium.
} 


\section{INSIDE THE HUBBLE RADIUS}

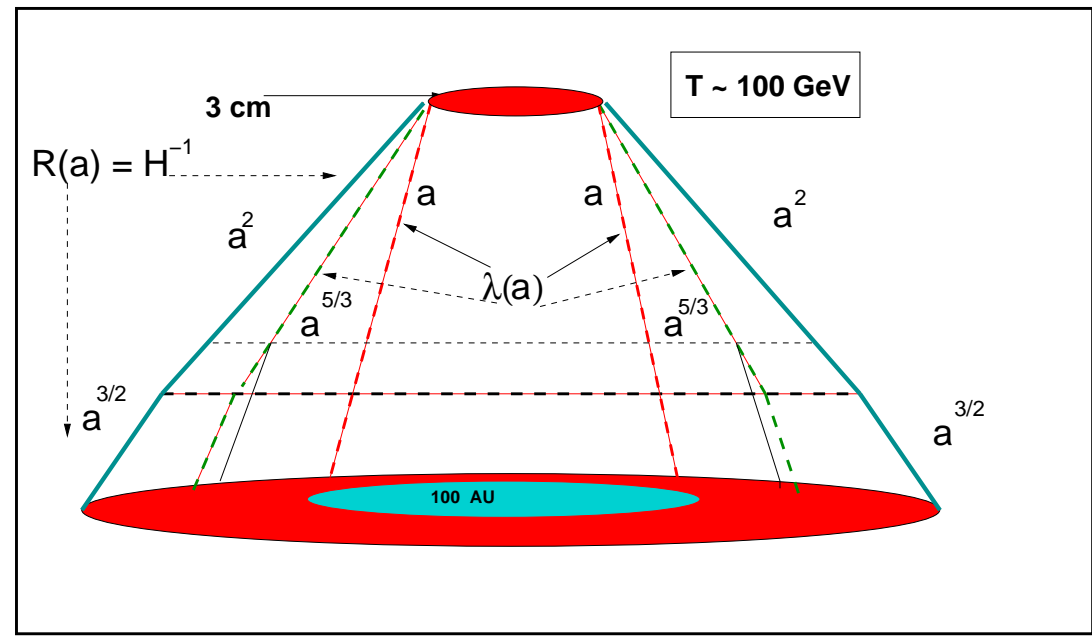

Figure 1: Evolution of the correlation scale for magnetic fields produced inside the Hubble radius. The horizontal thick dashed line marks the end of the radiation-dominated phase and the onset of the matter-dominated phase. The horizontal thin dashed line marks the moment of $e^{+}-e^{-}$annihilation (see also footnoote 2). The full (vertical) lines represent the evolution of the Hubble radius during the different stages of the life of the Universe. The dashed (vertical) lines illustrate the evolution of the correlation scale of the magnetic fields. In the absence of inverse cascade the evolution of the correlation scale is given by the (inner) vertical dashed lines. If inverse cascade takes place the evolution of the correlation scale is faster than the first power of the scale factor (for instance $a^{5 / 3}$ ) but always slower than the Hubble radius. 
the correlation scale of the magnetic field as a function of the scale factor. In Fig. 1 the horizontal dashed line simply marks the end of the radiation-dominated phase and the onset of the matter dominated phase: while above the dashed line the Hubble radius evolves as $a^{2}$ (where $a$ is the scale factor), below the dashed line the Hubble radius evolves as $a^{3 / 2}$.

We consider, for simplicity, a magnetic field whose typical correlation scale is as large as the Hubble radius at the electro-weak epoch when the temperature of the plasma was of the order of $100 \mathrm{GeV}$. If the correlation scale evolves as the scale factor, the Hubble radius at the electroweak epoch (roughly $3 \mathrm{~cm}$ ) projects today over a scale of the order of the astronomical unit. If inverse cascades are invoked, the correlation scale may grow, depending on the specific features of the cascade, up to 100 A.U. or even up to 100 pc. In both cases the final scale is too small if compared with the typical scale of the gravitational collapse of the proto-galaxy. In Fig. 1 a particular model for the evolution of the correlation scale $\lambda(a)$ has been reported ${ }^{3}$.

In the context of (conventional or unconventional) inflationary models, on the contrary, the correlation scale of the produced magnetic fields may be rather high. The physical picture is that during the inflationary phase the quantum fluctuations of the hypercharge field are amplified either through some coupling to the geometry or through the coupling to some other spectator field. Since during inflation the Hubble radius is roughly constant (see Fig. 2), the correlation scale evolves much faster than the Hubble radius itself and, therefore, large scale magnetic domains can naturally be obtained. Notice that, in Fig. 2 the (vertical) dashed lines illustrate the evolution of the Hubble radius (that is roughly constant during inflation) while the full line denotes the evolution of the correlation scale. Furthermore, the horizontal (dashed) lines mark, from top to bottom, the end of the inflationary phase and the onset of the matter-dominated phase. This phenomenon can be understood as the gauge counterpart of the super-adiabatic amplification of the scalar and tensor modes of the geometry. The main problem, in such a framework, is to get large amplitudes for scale of the order of the Mpc at the onset of gravitational collapse. Models where the gauge couplings are effectively dynamical (breaking, consequently, the Weyl invariance of the evolution equations of Abelian gauge modes) may provide rather intense magnetic fields.

The two extreme possibilities mentioned above may be sometimes combined. For instance, it can happen that magnetic fields are produced by super-adiabatic amplification of vacuum fluctuations during an inflationary stage of expansion. After exiting the horizon, the gauge modes will reenter at different moments all along the radiation and matter

\footnotetext{
${ }^{3}$ Notice, as it will be discussed later, that the inverse cascade lasts, in principle, only down to the time of $e^{+}--e^{-}$annihilation (see also thin dashed horizontal line in Fig. 1) since for temperatures smaller than $T_{e^{+}-e^{-}}$the Reynolds number drops below 1 . This is the result of the sudden drop in the number of charged particles that leads to a rather long mean free path for the photons.
} 
dominated epochs. The spectrum of the primordial gauge fields after reentry will not only be determined by the amplification mechanism but also on the plasma effects. As soon as the magnetic inhomogeneities reenter, some other physical process, taking place inside the Hubble radius, may be triggered by the presence of large scale magnetic fields. An example, in this context, is the production of topologically non-trivial configurations of the hypercharge field (hypermagnetic knots) from a stochastic background of hypercharge fields with vanishing helicity.

Since magnetic fields are produced over different length scales, it seems plausible to discuss their effect on the anisotropies of the Cosmic Microwave Background radiation (CMB). The finite values of the thermal and magnetic diffusivity scales forbids the presence of magnetic fields over length-scales that are very small in comparison with the size of the Hubble radius at a given epoch. On the other hand, dissipation is not effective in erasing the magnetic energy density over very large scales comparable with the Hubble radius itself.

Different topics are relevant for the interplay between large-scale magnetic fields and CMB physics. A simplified list of the main effects involving, simultaneously, large-scale magnetic fields and CMB physics must include:

- possible distortions of the CMB blackbody spectrum;

- possible induction of primary anisotropies;

- possible effects on the CMB polarization.

To this list it is appropriate to add another important topic that has to do with the subtraction of foregrounds. In fact, to disentangle the signal of the CMB anisotropies one has usually to subtract the emission of the galaxy. The synchrotron emission is indeed one of the foregrounds to be subtracted.

To estimate the effects of large-scale magnetic fields on CMB physics various approximations have been used through the years. Again, for sake of simplicity, it is useful to divide them into two broad classes:

- uniform field approximation;

- inhomogeneous field approximation.

In the uniform field approximation, the magnetic field is taken to be uniform and pointing along a specific direction in the sky. In this approximation (pioneered by Zeldovich in the sixties) the magnetic field slightly breaks the spatial isotropy of the background geometry. The break in the spatial isotropy can be easily connected with a difference in the propagation 


\section{SUPERADIABATIC AMPLIFICATION}

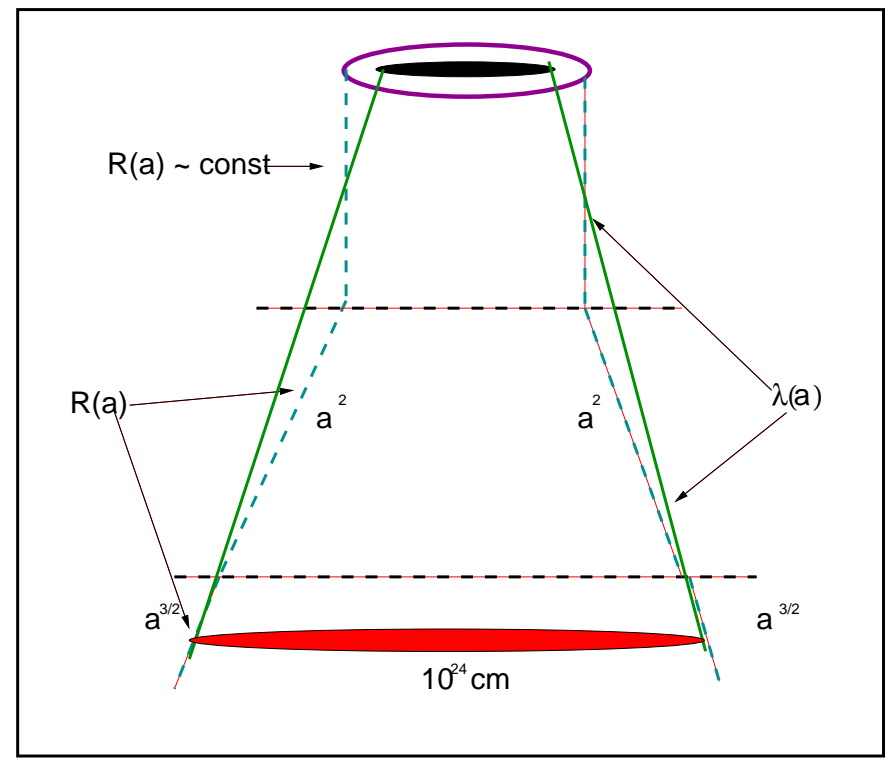

Figure 2: Evolution of the correlation scale if magnetic fields would be produced by superadiabatic amplification during a conventional inflationary phase. The dashed vertical lines denote, in the present figure, the evolution of the Hubble radius while the full line denotes the evolution of the correlation scale (typically selected to smaller than the Hubble radius during inflation).

of electromagnetic signals along different spatial directions. This phenomenon is ultimately responsible, in this context, for the anisotropy of the CMB.

In a complementary perspective, fully inhomogeneous large-scale magnetic fields do not break the spatial isotropy of the background geometry. This happens, for instance, when the magnetic field is fully inhomogeneous and characterized by its two-point function. In the uniform field approximation the resulting evolution equations are rather tractable and semianalytical estimates of various effects can be swiftly obtained. It is, however, problematic to imagine that a uniform magnetic field (oriented along a specific spatial direction) may be generated in the early Universe. The presence of a uniform field could be viewed as a remnant of the initial conditions of the evolution of the Universe.

Conversely, the fully inhomogenous approach is more realistic but also harder to implement analytically. An intermediate approach is to discuss the uniform field case supplemented by the appropriate fluctuations. In this approach the parameters describing the model are given by the uniform component of the magnetic field and by the two-point 
function of the fluctuations.

An important point to be borne in mind when discussing the effects of large-scale magnetic fields on CMB anisotropies concerns the suitable set of equations used to model the plasma effects. For the purposes of this paper we will confine ourselves to the two most practical sets of equations normally employed in this type of studies, namely

- a set of two-fluid plasma equations in curved space;

- an effective one-fluid (magnetohydrodynamical) description.

These two systems of equations are not equivalent for all the frequencies of the spectrum of plasma excitations. In particular, if we ought to address phenomena involving the propagation of electromagnetic disturbances in the plasma (as in the case of Faraday) rotation, the two fluid description (or even a kinetic Vlasov-Landau description) have to be employed. Magnetohydrodynamics (MHD) is a good effective description for sufficiently small frequencies in the spectrum of plasma excitations.

The plan of the present review is the following. In Section 2 the uniform field approximation will be reviewed. In Section 3 a two-fluid plasma description will be introduced. As an application, the Faraday effect in the uniform field approximation will be discussed. In Section 4, after discussing a one-fluid plasma description, fully inhomogeneous magnetic fields will be analyzed. Section 5 contains an introduction to the evolution of metric fluctuations in the presence of large-scale magnetic fields. In this context, the problem of initial conditions of CMB anisotropies will be reviewed in the presence of large-scale magnetic fields. The final part of Section 5 contains a summary of the numerical results obtained so far in the context of fully inhomogeneous magnetic fields. A brief collection of concluding remarks and future perspectives is collected in Section 6 .

\section{Uniform field approximation}

The uniform field approximation has a long history that could be traced back to the work of Zeldovich [1] (see also [2, 3]). Before the formulation of inflationary models the problem of the the initial conditions of Friedmann-Robertson-Walker models was debated. Among different options (early dominance of viscous effects, anisotropy in the expansion, etc.) a particularly interesting possibility was represented by a uniform background magnetic field oriented along a specific spatial direction. Indeed, close to a big-bang singularity the spatial gradients are sub-leading but the model can well by anisotropic [4, 5, 6]. In other words, close to the big bang, the homogeneous (but anisotropic) metric can fall in one of 


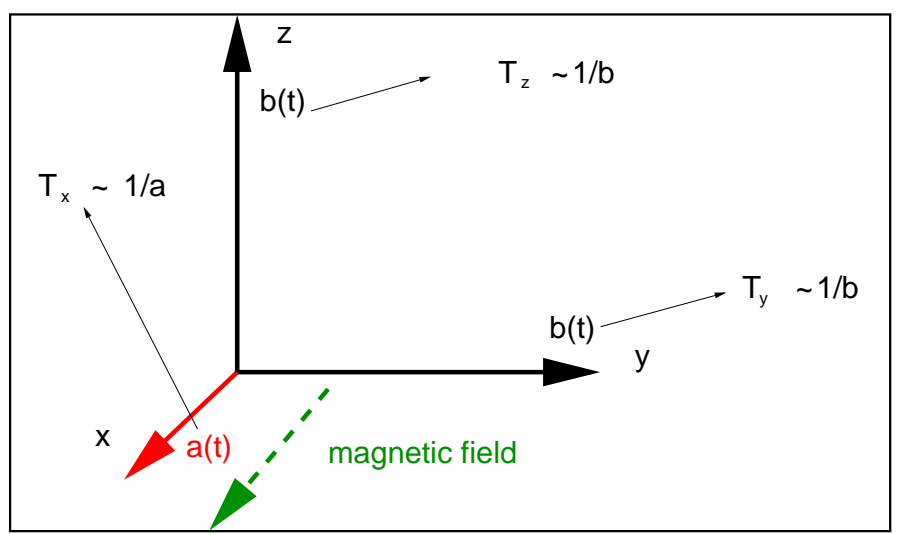

Figure 3: Uniform field approximation. A simplified geometrical configuration where the magnetic field is directed along the $\hat{x}$ axis.

the Bianchi classes [7] and a uniform magnetic field can fit into this scheme. During an inflationary phase the anisotropy decays exponentially and, therefore, we have to imagine that the (uniform) field considered in this section was created, for instance, close to the end of inflation. Since the work of Zeldovich [1, 2, the uniform field approximation has been exploited in various frameworks (see Refs. [8, 9, 10, 11, 12, 13] and references therein for a tentative list of theoretical studies).

Consider then, as an illustration, the case when the magnetic field is oriented along the $\hat{x}$ axis. Suppose also, for sake of simplicity, that the Universe is filled by a perfect barotropic fluid and that the spatial curvature vanishes. In this case the line element must account for the possibility of a different expansion rate along the $\hat{x}$ axis or along the $(\hat{y}, \hat{z})$ plane and may have the form

$$
d s^{2}=d t^{2}-a^{2}(t) d x^{2}-b^{2}(t)\left(d y^{2}+d z^{2}\right) .
$$

The metric (2.1) corresponds to a particular case of a Bianchi-type I metric. Uniform magnetic fields can be introduced also in other Bianchi metrics [8, 10, 11, 12]. The anisotropy in the expansion and the total expansion rate are, in this case,

$$
\mathcal{A}=\frac{H-F}{\Theta}, \quad \Theta=\frac{H+2 F}{3},
$$

where $H=\dot{a} / a$ and $F=\dot{b} / b$ are the expansion rates and the overdot denotes a derivation with respect to the cosmic time coordinate $t$.

In the model defined by Eq. (2.1) the propagation of an electromagnetic signal will be different along the $\hat{x}$ and $\hat{y}$ axes (see Fig. 3). Therefore, the temperature anisotropy will 
depend both on $\Theta$ and $\mathcal{A}$ :

$$
\frac{\Delta T}{T}=\frac{T_{x}-T_{y}}{T_{x}} \simeq-\int \mathcal{A} \Theta d t
$$

where we used that $T_{x}=T_{0} / a$ and $T_{y}=T_{0} / b$ together with the identities $a^{-1}=e^{-\int H d t}$ and $b^{-1}=e^{-\int F d t}$.

The quantities appearing in Eq. (2.3) can be estimated by studying the evolution of the anisotropic expansion and of the total expansion rate along the different stages of the life of the Universe. For this purpose it is practical to phrase Einstein equations directly in terms of $\mathcal{A}$ and $\Theta$. The procedure is rather simple since it suffices to write the extrinsic curvature in terms of $H$ and $F$. Then, by recalling the definitions of Eq. (2.2), the full set of Einstein equations and of the continuity equations can be written, respectively, as

$$
\begin{aligned}
& \dot{\Theta}+3 \Theta^{2}=\frac{8 \pi G}{3} \rho\left[\frac{2-3 \epsilon}{2}+q\right], \\
& \dot{\mathcal{A}} \Theta+\mathcal{A}\left(\dot{\Theta}+3 \Theta^{2}\right)=-16 \pi G \rho q, \\
& \dot{\rho}+\Theta(4+3 \epsilon) \rho=0, \\
& \dot{q}=3 q \Theta \epsilon+\frac{4}{3} q \Theta \mathcal{A},
\end{aligned}
$$

where the following conventions have been adopted

$$
w=\epsilon+\frac{1}{3}, \quad q=\frac{\rho_{\mathrm{B}}}{\rho} .
$$

In Eq. (2.8) $w$ is the barotropic index of the fluid background and

$$
\rho_{\mathrm{B}}=\frac{\mathcal{B}^{2}}{8 \pi}, \quad \dot{\rho}_{\mathrm{B}}+4 F \rho_{\mathrm{B}}=0 .
$$

In the case $\epsilon=0$ the barotropic index is exactly the one of radiation and Eqs. (2.4) (2.7) admit a solution where $\dot{\mathcal{A}} \simeq 0$ and $\Theta \simeq(2 t)^{-1}$. Inserting then Eq. (2.5) into Eq. (2.7) (and recalling that $\dot{\Theta}=-2 \Theta^{2}$ ) it is easy to obtain the evolution of $q(t)$, i.e.

$$
q(t)=\frac{q_{1}}{1+4 q_{1} \ln \left(\frac{t}{t_{1}}\right)}, \quad t \geq t_{1} .
$$

This means that in the case of a radiation background the anisotropy in the expansion is conserved and the ratio between radiation and magnetic energy densities is roughly constant (up to logarithmic corrections). For generalization of this analysis to more complicated situations involving different equations of state or various fluids and fields see, for instance, [8, 10, 13.

The property expressed by Eq. (2.10) is a consequence of the fact that both energymomentum tensors (i.e. the one of the background fluid and the one of the magnetic field) 
are traceless. Therefore, the evolution of $q(t)$ will be different if the background fluid is dominated by dusty sources. In the case of dusty matter Eq. (2.8) implies that $\epsilon=-1 / 3$. Therefore, an approximate solution of the system in this case is given by

$$
\Theta(t) \simeq \frac{2}{3 t}, \quad \mathcal{A}(t) \simeq-12 q_{\mathrm{eq}}\left(\frac{a_{\mathrm{eq}}}{a}\right), \quad q(t)=q_{\mathrm{eq}}\left(\frac{a_{\mathrm{eq}}}{a}\right) .
$$

By following the evolution of the system through equality up to decoupling the value of the anisotropy can then be roughly calculated to be

$$
\frac{\Delta T}{T} \simeq \frac{\mathcal{B}^{2}}{16 \pi \rho_{\gamma}} z_{\mathrm{dec}}
$$

By requiring that the temperature anisotropy is smaller than $10^{-5}$ we get in the case of $\Omega_{\mathrm{tot}}=1$

$$
\mathcal{B}_{0} \leq 2 \times 10^{-9} \text { Gauss, }
$$

where $\mathcal{B}_{0}$ refers to the value of the field at the present time. The logic leading to the limit reported in Eq. (2.13) has been followed, for instance, in Ref. 9].

A complementary way of describing the effect of a uniform magnetic field on the temperature anisotropies relies on the observation that, thanks to the breaking of spatial isotropy, the angular power spectrum will not depend only on the polar angle but also on the azimuthal angle. In fact, if spatial isotropy is unbroken, the angular power spectrum is customarily defined as

$$
C(\vartheta)=\left\langle\Delta_{\mathrm{I}}\left(\hat{n}_{1}, \tau_{0}\right) \Delta_{\mathrm{I}}\left(\hat{n}_{2}, \tau_{0}\right)\right\rangle
$$

where $\Delta_{\mathrm{I}}$ is the brightness perturbation in the intensity of the radiation field whose expansion in terms of spherical harmonics is

$$
\Delta_{\mathrm{I}}\left(\hat{n}, \tau_{0}\right)=\sum_{\ell=0}^{\infty} \sum_{m=-\ell}^{\ell} a_{\ell m} Y_{\ell m}(\hat{n}) .
$$

If the background space is isotropic, the ensemble average of $a_{\ell m}$ only depends upon $\ell$, not upon $m$, i.e.

$$
\left\langle a_{\ell m} a_{\ell^{\prime} m^{\prime}}^{*}\right\rangle=C_{\ell} \delta_{\ell \ell^{\prime}} \delta_{m m^{\prime}}
$$

Therefore, inserting Eq. (2.15) into Eq. (2.14), the addition theorem of spherical harmonics, i.e.

$$
P_{\ell}\left(\hat{n}_{1} \cdot \hat{n}_{2}\right)=\frac{4 \pi}{2 \ell+1} \sum_{m=-\ell}^{\ell} Y_{\ell m}^{*}\left(\hat{n}_{1}\right) Y_{\ell m}\left(\hat{n}_{2}\right),
$$

leads to the usual form of the angular power spectrum:

$$
C(\vartheta)=\left\langle\Delta_{\mathrm{I}}\left(\hat{n}_{1}, \tau_{0}\right) \Delta_{\mathrm{I}}\left(\hat{n}_{2}, \tau_{0}\right)\right\rangle \equiv \frac{1}{4 \pi} \sum_{\ell}(2 \ell+1) C_{\ell} P_{\ell}(\vartheta) .
$$


where $\vartheta=\hat{n}_{1} \cdot \hat{n}_{2}$.

If spatial isotropy is slightly broken (as in the case when a uniform magnetic field is present), Eq. (2.16) is not justified since the ensemble average of the $a_{\ell m}$ should also depend upon $m$ and not only upon $\ell$. Indeed, it can be shown that when a uniform magnetic field is present, the average of temperature fluctuations in two directions of the sky can be expressed as

$$
\begin{aligned}
& \left\langle\Delta_{\mathrm{I}}\left(\hat{n}_{1}, \tau_{0}\right) \Delta\left(\hat{n}_{2}, \tau_{0}\right)\right\rangle=\sum_{\ell, m} C_{\ell}(m) Y_{\ell, m}\left(\hat{n}_{1}\right) Y_{\ell, m}^{*}\left(\hat{n}_{2}\right) \\
& +\sum_{\ell, m} D_{\ell}(m)\left[Y_{\ell+1, m}\left(\hat{n}_{1}\right) Y_{\ell-1, m}^{*}\left(\hat{n}_{2}\right)+Y_{\ell-1, m}\left(\hat{n}_{1}\right) Y_{\ell+1, m}^{*}\left(\hat{n}_{2}\right)\right],
\end{aligned}
$$

where both $C_{\ell}(m)$ and $D_{\ell}(m)$ depend upon the (uniform) magnetic field intensity. From Eq. (2.19) it is apparent that the presence of a magnetic field with uniform component induces off-diagonal correlations in multipole space between a given $\ell$ and multipoles $\ell \pm 2$. The breaking of spatial isotropy in the CMB maps can be constrained, for instance, from WMAP data .

This analysis has been recently performed in [14] (see also [15]). The constraints on the off-diagonal correlations in multipole space imply a bound on the (present) uniform component of the magnetic field intensity

$$
\mathcal{B}_{0} \leq 1.7 \times 10^{-9} \mathrm{G}
$$

It should be mentioned that the bound of Eq. (2.20) has been derived by taking into account also the fluctuations of the magnetic field around the uniform configuration. The presence of the fluctuations of a uniform configuration introduces extra parameters (like the spectral index of the fluctuations) that make the allowed region in the parameter space effectively two-dimensional. Various studies assume a (uniform) background field and consider the possible fluctuations of such a configuration (see for instance [16, 17, 18] and references therein). In particular, in Ref. [17] the magnetic field has been parametrized in terms of a uniform component directed along a specific axis and a tangled component. The Alfénwaves modes associated with this configuration may induce a small rotational perturbation in the last scattering surface.

In [19] this analysis has been performed in a non-relativistic approximation where the scalar perturbations of the geometry obey linearized Newtonian equations of motion. The magnetic field has been assumed to be uniform (but supplemented with the appropriate fluctuations). In this framework, it has been argued that the effect of the presence of the (uniform) magnetic can be phrased into an effective renormalization of the speed of sound of the baryons (see also [20]). 


\section{Two-fluid plasma description and CMB polarization}

Right before decoupling the value of the Debye screening length is much larger than the electron and photon mean free paths. In fact, the Debye length scale can be written as

$$
\lambda_{\mathrm{D}}=\sqrt{\frac{T_{\mathrm{ei}}}{8 \pi e^{2} n_{0}}} \simeq 10\left(\frac{n_{0}}{10^{3} \mathrm{~cm}^{-3}}\right)^{-1 / 2}\left(\frac{T_{\mathrm{ei}}}{0.3 \mathrm{eV}}\right)^{1 / 2} \mathrm{~cm} .
$$

where $n_{0} \simeq n_{\mathrm{e}} \simeq n_{\mathrm{i}}$ is the mean electron-ion density and $T_{\mathrm{ei}}$ is the electron-ion temperature. For length scales larger than $\lambda_{\mathrm{D}}$, the plasma is globally neutral. For typical values of the parameters in Eq. (3.1), $\lambda_{\mathrm{D}}$ is of the order of $10 \mathrm{~cm}$ around recombination while the electron and photon mean free paths are much larger, i.e.

$$
\ell_{\mathrm{e}} \simeq 5.7 \times 10^{7} \mathrm{~cm}, \quad \ell_{\gamma} \simeq 10^{4}(1+z)^{-2}\left(\Omega_{\mathrm{b}} h_{0}^{2}\right)^{-1} \mathrm{Mpc},
$$

where $z=a_{0} / a-1$ is the redshift, $\Omega_{\mathrm{b}}$ the baryon fraction of the critical energy density and $h_{0}$ the indetermination in the Hubble parameter.

The other set of quantities relevant for the determination of the appropriate system of equations is given by the plasma and Larmor frequencies of electrons and ions, i.e.

$$
\begin{aligned}
& \omega_{\mathrm{pe}} \simeq 2\left(\frac{n_{e}}{10^{3} \mathrm{~cm}^{-3}}\right)^{1 / 2} \mathrm{MHz}, \quad \omega_{\mathrm{Be}} \simeq 18.08\left(\frac{\mathcal{B}}{10^{-3} \mathrm{G}}\right) \mathrm{kHz}, \\
& \omega_{\mathrm{pi}} \simeq 40\left(\frac{n_{e}}{10^{3} \mathrm{~cm}^{-3}}\right)^{1 / 2} \mathrm{kHz}, \quad \omega_{\mathrm{Bi}} \simeq 9.66\left(\frac{\mathcal{B}}{10^{-3} \mathrm{G}}\right) \mathrm{Hz} .
\end{aligned}
$$

Note that the typical value of $\mathcal{B}$ around decoupling reported in Eqs. (3.3) and (3.4) corresponds, today, to a magnetic field of the order of $10^{-9}-10^{-10} \mathrm{G}$.

If we are now interested in the propagation of electromagnetic disturbances with frequencies larger than the plasma and Larmor frequencies, then it is clear that a two-fluid plasma description should be adopted. For some classes of problems it will also be useful to employ directly a kinetic (Vlasov-Landau [21, 22, 23]) description. If, on the contrary, we would be interested in the spectrum of plasma excitations for $\omega<\omega_{\mathrm{p}}$, then effective (one-fluid) descriptions (like MHD [24]) can be usefully employed. This second regime will be addressed in Section 4 .

The two-fluid plasma equations in curved space-time will now be discussed and treated in analogy with the well-known flat-space case [25]. Let us consider, for simplicity a conformally flat geometry of Friedmann-Robertson-Walker (FRW) type:

$$
d s^{2}=a^{2}(\tau)\left[d \tau^{2}-d \vec{x}^{2}\right] .
$$

The following two equations account for the continuity of electrons and ions charge densities

$$
\begin{aligned}
& n_{\mathrm{e}}^{\prime}+3 w_{\mathrm{e}} \mathcal{H} n_{\mathrm{e}}+\left(w_{\mathrm{e}}+1\right) \vec{\nabla} \cdot\left(n_{\mathrm{e}} \vec{v}_{\mathrm{e}}\right)=0, \\
& n_{\mathrm{i}}^{\prime}+3 w_{\mathrm{i}} \mathcal{H} n_{\mathrm{i}}+\left(w_{\mathrm{i}}+1\right) \vec{\nabla} \cdot\left(n_{\mathrm{i}} \vec{v}_{\mathrm{i}}\right)=0,
\end{aligned}
$$


where the prime denotes a derivation with respect to the conformal time coordinate $\tau$ while the other quantities appearing in Eqs. (3.6) and (3.7) are

$$
\mathcal{H}=a^{\prime} / a, \quad n_{\mathrm{e}}=a^{3} \tilde{n}_{\mathrm{e}}, \quad n_{\mathrm{i}}=a^{3} \tilde{n}_{\mathrm{i}} .
$$

In Eqs. (3.6) and (3.7) $w_{\mathrm{e}}$ and $w_{\mathrm{i}}$ are the barotropic indices for the electron and ion fluids. Both electrons and ions are non-relativistic (for the ion mass, in the numerical estimates, we take $m_{\mathrm{i}} \simeq m_{\mathrm{p}}$ where $m_{\mathrm{p}}$ denotes the proton mass). Hence the barotropic indices $w_{\mathrm{e}}$ and $w_{\mathrm{i}}$ will be close to zero. In fact, the energy and pressure densities of an ideal electronic gas are given by

$$
\rho_{\mathrm{e}}=n_{\mathrm{e}}\left(m_{\mathrm{e}}+\frac{3}{2} T_{\mathrm{e}}\right), \quad p_{\mathrm{e}}=n_{\mathrm{e}} T_{\mathrm{e}}
$$

and since $w_{\mathrm{e}, \mathrm{i}}=T_{\mathrm{e}, \mathrm{i}} / m_{\mathrm{e}, \mathrm{i}}$, we will also have $w_{\mathrm{e}, \mathrm{i}} \ll 1$ as far as $T_{\mathrm{e}, \mathrm{i}} \ll m_{\text {,ie. }}$. Recalling that $\rho_{\mathrm{e}}=m_{\mathrm{e}} n_{\mathrm{e}}$ and $\rho_{\mathrm{i}}=m_{\mathrm{i}} n_{\mathrm{i}}$, the momentum conservation equations are

$$
\begin{aligned}
& \rho_{\mathrm{e}}\left[\vec{v}_{\mathrm{e}}^{\prime}+\mathcal{H} \vec{v}_{\mathrm{e}}+\left(\vec{v}_{\mathrm{e}} \cdot \vec{\nabla}\right) \vec{v}_{\mathrm{e}}\right]=-n_{\mathrm{e}} e\left(\vec{E}+\frac{\vec{v}_{\mathrm{e}}}{c} \times \vec{B}\right), \\
& \rho_{\mathrm{i}}\left[\vec{v}_{\mathrm{i}}^{\prime}+\mathcal{H} \vec{v}_{\mathrm{i}}+\left(\vec{v}_{\mathrm{i}} \cdot \vec{\nabla}\right) \vec{v}_{\mathrm{i}}\right]=n_{\mathrm{i}} e\left(\vec{E}+\frac{\vec{v}_{\mathrm{i}}}{c} \times \vec{B}\right),
\end{aligned}
$$

where $\vec{E}=a^{2} \overrightarrow{\mathcal{E}}$ and $\vec{B}=a^{2} \overrightarrow{\mathcal{B}}$ are the conformally rescaled electromagnetic fields obeying the following set of equations:

$$
\begin{aligned}
& \vec{\nabla} \cdot \vec{E}=4 \pi e\left(n_{\mathrm{i}}-n_{\mathrm{e}}\right), \\
& \vec{\nabla} \cdot \vec{B}=0, \quad \vec{\nabla} \times \vec{E}=-\frac{1}{c} \vec{B}^{\prime}, \\
& \vec{\nabla} \times \vec{B}=\frac{1}{c} \vec{E}^{\prime}+\frac{4 \pi e}{c}\left(n_{\mathrm{i}} \vec{v}_{\mathrm{i}}-n_{\mathrm{e}} \vec{v}_{e}\right) .
\end{aligned}
$$

The dispersion relations relevant for the propagation of electromagnetic waves with $\omega>\omega_{\mathrm{p}}$ can be found, after some algebra, in the case of a uniform magnetic field directed, for instance, along the $\hat{z}$ axis ${ }^{4}$.

Equations (3.6) and (3.7) can be first linearized around a globally neutral configuration, i.e.

$$
n_{\mathrm{e}}(\tau, \vec{x})=n_{0}+\delta n_{\mathrm{e}}(\tau, \vec{x}), \quad n_{\mathrm{i}}(\tau, \vec{x})=n_{0}+\delta n_{\mathrm{i}}(\tau, \vec{x}),
$$

where $n_{0}$ is the common electron and ion density. The remaining equations can be also linearized around the uniformly magnetized configuration:

$$
\begin{aligned}
& \vec{B}(\eta, \vec{x})=\vec{B}_{0}+\delta \vec{B}(\eta, \vec{x}), \\
& \vec{v}_{\mathrm{e}, \mathrm{i}}(\eta, \vec{x})=\delta \vec{v}_{\mathrm{e}, \mathrm{i}}(\eta, \vec{x}), \quad \vec{E}(\eta, \vec{x})=\delta \vec{E}(\eta, \vec{x}) .
\end{aligned}
$$

\footnotetext{
${ }^{4}$ In principle we should allow a different expansion rate along the $(\hat{x}, \hat{y})$ plane and along the $\hat{z}$ axis, as discussed in the previous Section (see, for instance, Eq. 2.1). This is not strictly necessary for the derivation of the dispersion relations. While the anisotropic expansion caused by a uniform magnetic field affects the primary anisotropies, the dispersion relations are weakly affected by the curved space corrections. This statement will be explicitly verified in our case.
} 
The linearized equations around the uniformly magnetized background can be solved [26]. The dielectric tensor can then be obtained and it has the following general form [26]:

$$
\bar{\epsilon}(\omega, \alpha)=\left(\begin{array}{ccc}
\epsilon_{1}(\omega, \alpha) & i \epsilon_{2}(\omega, \alpha) & 0 \\
-i \epsilon_{2}(\omega, \alpha) & \epsilon_{1}(\omega, \alpha) & 0 \\
0 & 0 & \epsilon_{\|}(\omega, \alpha)
\end{array}\right)
$$

where

$$
\begin{aligned}
& \epsilon_{\|}(\omega, \alpha)=1-\frac{\omega_{\mathrm{p}, \mathrm{i}}^{2}}{\omega^{2}(1+\alpha)}-\frac{\omega_{\mathrm{p}, \mathrm{e}}^{2}}{\omega^{2}(1+\alpha)} \\
& \epsilon_{1}(\omega, \alpha)=1-\frac{\omega_{\mathrm{pi}}^{2}(\alpha+1)}{\omega^{2}(\alpha+1)^{2}-\omega_{\mathrm{Bi}}^{2}}-\frac{\omega_{\mathrm{pe}}^{2}(\alpha+1)}{\omega^{2}(\alpha+1)^{2}-\omega_{\mathrm{Be}}^{2}}, \\
& \epsilon_{2}(\omega, \alpha)=\frac{\omega_{\mathrm{Be}}}{\omega} \frac{\omega_{\mathrm{p} \mathrm{e}}^{2}}{\omega^{2}(\alpha+1)^{2}-\omega_{\mathrm{Be}}^{2}}-\frac{\omega_{\mathrm{Bi}}}{\omega} \frac{\omega_{\mathrm{pi}}^{2}}{\omega^{2}(\alpha+1)^{2}-\omega_{\mathrm{Bi}}^{2}} .
\end{aligned}
$$

The quantity $\epsilon_{\|}(\omega, \alpha)$ denotes the dielectric constant parallel to the direction of the uniform component of the magnetic field intensity while $\epsilon_{1}(\omega, \alpha)$ and $\epsilon_{2}(\omega, \alpha)$ denote the dielectric constants determined by the motion of charged particles orthogonal to the direction of the uniform magnetic field. If the uniform magnetic field vanishes, we can obtain the dielectric tensor of the cold plasma by formally taking the limit $\epsilon_{2}(\omega, \alpha) \rightarrow 0$ and $\epsilon_{1}(\omega, \alpha) \rightarrow \epsilon_{\|}(\omega, \alpha)$. It is then clear that in the absence of magnetic field the only scales entering the dispersion relations are the plasma frequencies of electrons and ions. The quantity $\alpha$ appearing in the dielectric tensor is defined as $\alpha=i \mathcal{H} / \omega$ and is the lowest order correction arising in curved space. This correction is negligible for the present purposes since it is of the order of the ratio between the Hubble radius at the decoupling and the inverse of the frequency of propagation. Recalling that $H_{\mathrm{dec}} \simeq 5.6 \times 10^{12}\left(\Omega_{0} h_{0}^{2}\right)^{-1 / 2}$ sec and that $\omega \gg 100 \mathrm{MHz}$, we have that $|\alpha|<10^{-22}$.

To derive the dispersion relations it is appropriate to fix the coordinate system: for instance we can set $k_{x}=0$ and $k_{y}=k \sin \theta, k_{z}=k \cos \theta$ with the magnetic field oriented along the $\hat{z}$ direction. Introducing the refraction index $n$ and recalling that $k=\omega / v=n \omega / c$ (where $v$ is the phase velocity) the dispersion relations are determined from the solution of the following equation:

$$
s^{2}(\theta)\left(\frac{1}{\epsilon_{\|}}-\frac{1}{n^{2}}\right)\left[\frac{1}{n^{2}}-\frac{1}{2}\left(\frac{1}{\epsilon_{\mathrm{L}}}+\frac{1}{\epsilon_{\mathrm{R}}}\right)\right]-c^{2}(\theta)\left(\frac{1}{n^{2}}-\frac{1}{\epsilon_{\mathrm{L}}}\right)\left(\frac{1}{n^{2}}-\frac{1}{\epsilon_{\mathrm{R}}}\right)=0,
$$

where $c(\theta)=\cos \theta$ and $s(\theta)=\sin \theta$. In Eq. (3.21) the following linear combinations have been also introduced:

$$
\begin{aligned}
& \epsilon_{\mathrm{R}}=\epsilon_{1}+\epsilon_{2}=1-\frac{\omega_{\mathrm{pi}}^{2}}{\omega\left[\omega(\alpha+1)-\omega_{\mathrm{Bi}}\right]}-\frac{\omega_{\mathrm{pe}}^{2}}{\omega\left[\omega(\alpha+1)+\omega_{\mathrm{B}}\right]}, \\
& \epsilon_{\mathrm{L}}=\epsilon_{1}-\epsilon_{2}=1-\frac{\omega_{\mathrm{pe}}^{2}}{\omega\left[\omega(\alpha+1)-\omega_{\mathrm{Be}}\right]}-\frac{\omega_{\mathrm{pi}}^{2}}{\omega\left[\omega(\alpha+1)+\omega_{\mathrm{Bi}}\right]} .
\end{aligned}
$$


The quantity $\epsilon_{\mathrm{R}}$ and $\epsilon_{\mathrm{L}}$ are the dielectric constants appropriate for the propagation of the two (left and right) circular polarizations. The dispersion relations for the propagation of electromagnetic disturbances parallel (i.e. $\theta=0$ ) and orthogonal (i.e. $\theta=\pi / 2$ ) to the uniform component of the magnetic field can then be obtained from Eq. (3.21):

$$
\begin{array}{ll}
\left(n^{2}-\epsilon_{\mathrm{R}}\right)\left(n^{2}-\epsilon_{\mathrm{L}}\right)=0, & \theta=0, \\
\left(n^{2}-\epsilon_{\|}\right)\left[n^{2}\left(\epsilon_{\mathrm{L}}+\epsilon_{\mathrm{R}}\right)-2 \epsilon_{\mathrm{L}} \epsilon_{\mathrm{R}}\right]=0, & \theta=\frac{\pi}{2} .
\end{array}
$$

Equation (3.24) gives the usual dispersion relations for the two circular polarizations of the electromagnetic wave, i.e. $n^{2}=\epsilon_{\mathrm{R}}$ and $n^{2}=\epsilon_{\mathrm{L}}$, while Eq. (3.25) gives those for the "ordinary" (i.e. $n^{2}=\epsilon_{\|}$) and "extraordinary" (i.e. $n^{2}=2 \epsilon_{\mathrm{R}} \epsilon_{\mathrm{L}} /\left(\epsilon_{\mathrm{R}}+\epsilon_{\mathrm{L}}\right)$ ) plasma waves.

Consider now an electromagnetic wave that is linearly polarized. A linearly polarized electromagnetic wave can be always seen as the sum of two circularly polarized waves. Now the left and right polarizations have different phase velocities and, therefore, after the waves have travelled a distance $\Delta z$ in a (cold) magnetized plasma the resulting phase difference will be

$$
\Delta \Phi=\frac{\omega}{2 c}\left[\sqrt{\epsilon_{\mathrm{R}}}-\sqrt{\epsilon_{\mathrm{L}}}\right] \Delta z
$$

Equation (3.26) has been derived using two physical assumptions

- the plasma is cold, i.e. the temperature of the electrons does not enter the dispersion relations;

- there are no other sources leading to a rotation of linearly polarized radiation.

Let us now see what happens by relaxing these two assumptions. The adoption of a cold (as opposed to warm) plasma description means that the temperature of the electrons has been taken to be effectively zero. The effects arising from the finite temperature of the electrons do not modify the leading result obtained in the context of the cold plasma theory. The derivation of the dispersion relations in the case of a warm plasma can be performed, for instance, within a kinetic approach where it can be shown, following the same calculation discussed in the flat space case [27], that the first correction to the leading cold plasma calculation can be recast in an effective redefinition of the plasma frequency for the electrons, namely $\omega_{\mathrm{pe}} \rightarrow \frac{4 \pi n_{0} e^{2}}{m_{e} \gamma}$ where $\gamma=\left(1-\left\langle v^{2}\right\rangle\right)^{-1 / 2}$ and $\left\langle v^{2}\right\rangle$ is the thermal average of the electron velocity.

Equation (3.26) has been derived under the assumption that the only source of birefringence is represented by a uniform magnetic field. This is certainly legitimate in a standard electromagnetic context. There could be however other sources of cosmological birefringence leading to a generalized expression of Faraday rotation. In particular let us consider 
the case when, in the plasma, a dynamical pseudo-scalar field is present together with a magnetic field. This situation may easily arise in the case of a quintessential background with pseudo-scalar coupling to electromagnetism described by an action

$$
S_{\sigma}=\int d^{4} x \sqrt{-g}\left[\frac{1}{2} g^{\alpha \beta} \partial_{\alpha} \sigma \partial_{\beta} \sigma-W(\sigma)+\frac{\beta}{4 M} F_{\alpha \beta} \tilde{F}^{\alpha \beta}\right],
$$

where $F_{\alpha \beta}$ and $\tilde{F}^{\alpha \beta}$ are the Maxwell field strength and its dual. The coupling of electromagnetism to the field $\sigma$ changes the form of Eqs. (3.12), (3.13) and (3.14). The resulting system of equations can be discussed in the presence both of a uniform magnetic field and of a cold (or warm) plasma. This calculation has been performed in Ref. [26]. While Eqs. (3.13) do not change, the generalization of Eqs. (3.12) and (3.14) becomes:

$$
\begin{aligned}
& \vec{\nabla} \cdot \vec{E}=4 \pi e\left(n_{\mathrm{i}}-n_{\mathrm{e}}\right)+\frac{\beta}{M} \vec{\nabla} \sigma \cdot \vec{B}, \\
& \vec{\nabla} \times \vec{B}=\frac{1}{c} \vec{E}^{\prime}-\frac{\beta}{M}\left[\sigma^{\prime} \vec{B}+\vec{\nabla} \sigma \times \vec{E}\right]+\frac{4 \pi e}{c}\left(n_{\mathrm{i}} \overrightarrow{\mathrm{v}}_{\mathrm{i}}-n_{\mathrm{e}} \vec{v}_{e}\right) .
\end{aligned}
$$

To understand the rationale of the physical difference induced by the presence of $\sigma$ consider, indeed, the case when $\nabla \sigma=0$ and the charge and current densities are vanishing. In this case, taking the curl of Eq. (3.29) and using the second of Eqs. (3.13) we obtain the following equation

$$
\vec{B}^{\prime \prime}-c^{2} \nabla^{2} \vec{B}+\frac{\beta}{M} c \sigma^{\prime} \vec{\nabla} \times \vec{B}=0 .
$$

Equation (3.30) implies that the two polarizations of the magnetic field are mixed as a consequence of the time-dependence of $\sigma$. If the plasma contribution is included, Eq. (3.21) is modified [26]. Consequently, the dispersion relations given in Eqs. (3.24) and (3.25) are also modified:

$$
\begin{aligned}
& \left(n^{2}-\frac{\omega_{\sigma}}{\omega} n-\epsilon_{\mathrm{R}}\right)\left(n^{2}+\frac{\omega_{\sigma}}{\omega} n-\epsilon_{\mathrm{L}}\right)=0, \quad \theta=0, \\
& n^{4}-\left[2 \frac{\epsilon_{\mathrm{L}} \epsilon_{\mathrm{R}}}{\epsilon_{\mathrm{L}}+\epsilon_{\mathrm{R}}}+\epsilon_{\|}+\left(\frac{\omega_{\sigma}}{\omega}\right)^{2}\right] n^{2}+2 \frac{\epsilon_{\|} \epsilon_{\mathrm{L}} \epsilon_{\mathrm{R}}}{\epsilon_{\mathrm{R}}+\epsilon_{\mathrm{L}}}=0, \quad \theta=\frac{\pi}{2} .
\end{aligned}
$$

The presence of the quintessence field introduces a further frequency scale into the problem, namely $\omega_{\sigma}=c(\beta / M) \sigma^{\prime}$. If the quintessence field dominates today (or between redshifts 0 and 3), $\sigma\left(t_{0}\right) \sim \Lambda^{2} / m$ where, typically, $\Lambda \sim 10^{-3} \mathrm{eV}$, and $m \sim 10^{-33} \mathrm{eV}$. The value of $\dot{\sigma}$ can be estimated, and it is, today, $\dot{\sigma}\left(t_{0}\right) \simeq m^{2} / H_{0}$. Hence, recalling that prior to quintessential dominance, $\dot{\sigma}$ scales as $a^{-3}$, from the previous expressions

$$
\dot{\sigma}_{\mathrm{dec}} \simeq\left(\frac{H_{\mathrm{dec}}}{H_{0}}\right)^{2} \frac{m \Lambda^{2}}{H_{0}}, \quad \omega_{\sigma}=\beta\left(\frac{M}{M_{\mathrm{P}}}\right) \times 10^{-6} \mathrm{~Hz},
$$

where the values of $\Lambda$ and $m$ are the ones discussed above with $M \simeq M_{\mathrm{P}}$. The value of $\beta$ is rather uncertain and a conservative limit from radio-astronomical analyses would imply 
$\beta \lesssim 10^{-3}$ for $0 \lesssim z \lesssim 1[28,29$. Notice that the action (3.27) can also be relevant in a class of baryogenesis models [30, 31] (see also [32]).

From Eq. (3.31) the generalized Faraday rotation experienced by the linearly polarized CMB travelling parallel to the magnetic field direction can be obtained as

$$
\Delta \Phi=\frac{\omega}{2 c}\left[\frac{\omega_{\sigma}}{\omega}+\sqrt{\frac{1}{2}\left(\frac{\omega_{\sigma}}{\omega}\right)^{2}+\epsilon_{\mathrm{R}}}-\sqrt{\frac{1}{2}\left(\frac{\omega_{\sigma}}{\omega}\right)^{2}+\epsilon_{\mathrm{L}}}\right] \Delta z .
$$

This expression includes, simultaneously, the birefringence produced by the evolution of the pseudo-scalar field $\sigma$ and the rotation induced by the finite value of the magnetic field intensity. In Ref. 34] the effect of parity-violating interactions on CMB polarization has been recently discussed in the absence of a magnetic field. A similar analysis was presented in [35] (see also [36]). In the absence of magnetic field, Eq. (3.34) leads to a $\Delta \Phi$ that is frequency independent. On the contrary, if the contribution of the pseudo-scalar field $\sigma$ is negligible, then $\Delta \Phi$ decreases at high frequencies as $1 / \omega^{2}$. In the intermediate situation more complicated dependences of $\Delta \Phi$ upon the frequency may be envisaged. This aspect answers the second question raised after Eq. (3.26): on top of the magnetic fields there are different sources affecting $\Delta \Phi$. However, the characteristic frequency dependence of the different signals may allow, at least in principle, to disentangle different sources of birefringence.

\subsection{Magnetized Faraday effect at decoupling}

Let us now assume, for simplicity, that the only source for $\Delta \Phi$ is represented by a uniform magnetic field. Then, recalling the hierarchy between the plasma, Larmor and propagation frequencies, i.e. Eqs. (3.3) and (3.4), Eq. (3.26) can be expanded for

$$
\left|\omega_{\mathrm{p}, \mathrm{e}, \mathrm{i}} / \omega\right| \ll 1, \quad\left|\omega_{\mathrm{B}, \mathrm{e}, \mathrm{i}} / \omega\right| \ll 1 .
$$

Thus, from Eq. (3.26), we will have:

$$
\Delta \Phi \simeq \frac{\omega}{2 c}\left\{\sqrt{1-\left(\frac{\omega_{\mathrm{p} \mathrm{e}}}{\omega}\right)^{2}\left[1-\frac{\omega_{\mathrm{B} \mathrm{e}}}{\omega}\right]}-\sqrt{1-\left(\frac{\omega_{\mathrm{p} \mathrm{e}}}{\omega}\right)^{2}\left[1+\frac{\omega_{\mathrm{B} \mathrm{e}}}{\omega}\right]}\right\} \Delta z,
$$

where, by virtue of Eqs. (3.3) and (3.4) the sub-leading contribution of the ions has been neglected. In some simplified treatments this approximation is made from the very beginning by defining two generalized refraction indices $n_{\mathrm{R}}$ and $n_{\mathrm{L}}$ :

$$
n_{\mathrm{R}, \mathrm{L}}=\sqrt{\epsilon_{\mathrm{R}, \mathrm{L}}}=1-\frac{\omega_{\mathrm{p}, \mathrm{e}}^{2}}{\omega\left(\omega \pm \omega_{\mathrm{B}, \mathrm{e}}\right)}
$$


( the plus and minus signs correspond, respectively, to $\mathrm{R}$ and $\mathrm{L}^{5}$ )

A last expansion for $\left|\omega_{\mathrm{B}, \mathrm{e}} / \omega\right| \ll 1$ and for $\left|\omega_{\mathrm{p}, \mathrm{e}} / \omega\right| \ll 1$ brings Eq. (3.36) to the standard form, i.e.

$$
\Delta \Phi=\frac{1}{2 c}\left(\frac{\omega_{\mathrm{p}, \mathrm{e}}}{\omega}\right)^{2} \omega_{\mathrm{B}, \mathrm{e}} \Delta z .
$$

From Eq. (3.38), by choosing a generic propagation direction, we can also derive the Faraday rotation rate, i.e.

$$
\frac{d \Phi}{d t}=\frac{e^{3} x_{\mathrm{e}} n_{0}}{2 \pi c m_{\mathrm{e}}^{2} \nu^{2}} \vec{B} \cdot \hat{n}
$$

where the inonization fraction $x_{\mathrm{e}}$ has been introduced by replacing $n_{0} \rightarrow x_{\mathrm{e}} n_{0}$ and where we used, from Eq. (3.38), that $d z=c d t$. In Eq. (3.39), $\vec{B} \cdot \hat{n}$ denotes the projection of the (uniform) magnetic field intensity along the direction of propagation of the linearly polarized radiation with frequency $\nu=\omega / 2 \pi ; t$ is, as usual, the cosmic time coordinate related to the conformal time coordinate $\tau$ as $a(\tau) d \tau=d t$.

For some applications it is sometimes useful to write Eq. (3.39) in apparently different forms. Recalling the definition of differential optical depth

$$
\epsilon^{\prime}=x_{\mathrm{e}} n_{0} \sigma_{\mathrm{T}} \frac{a}{a_{0}}=\frac{x_{\mathrm{e}} n_{0} \sigma_{\mathrm{T}}}{z+1} .
$$

Defining with $\tau_{0}$ the conformal time at which the signal is received, the optical depth will then be

$$
\epsilon\left(\tau, \tau_{0}\right)=\int_{\tau}^{\tau_{0}} x_{\mathrm{e}} n_{0} \sigma_{\mathrm{T}} \frac{a}{a_{0}} d \tau
$$

while the visibility function ${ }^{6}$ is

$$
\mathcal{K}(\tau)=\epsilon^{\prime} e^{-\epsilon\left(\tau, \tau_{0}\right)} .
$$

Equation (3.42) gives the probability that a CMB photon was last scattered between $\tau$ and $\tau+d \tau$ (usually in the literature $\mathcal{K}(\tau)$ is denoted with $g(\tau)$ ).

Using Eq. (3.41), Eq. (3.39) can be written as

$$
\Omega_{\Phi}=\Phi^{\prime}=\frac{d \Phi}{d \tau}=\frac{3 c^{3} \epsilon^{\prime}}{16 \pi^{2} e} \vec{B} \cdot \hat{n}
$$

where the explicit expression of the Thompson cross section in terms of the classical radius of the electron has been used, i.e.

$$
\sigma_{\mathrm{T}}=\frac{8 \pi}{3} r_{0}^{2}, \quad r_{0}=\frac{e^{2}}{m_{\mathrm{e}} c^{2}}
$$

\footnotetext{
${ }^{5}$ Recalling that $\omega=k c / n_{\mathrm{R}, \mathrm{L}}$, by compute $\partial \omega / \partial k$ we can easily get, from Eq. (3.37), and approximate expression of the group velocity.

${ }^{6}$ Recently the possible modifications induced by large-scale magnetic fields on the thermal history of the Universe during the "dark age" beteween $z \sim 1000$ and $z \sim 10$ has been studied in [33].
} 
Equation (3.43) can be also written in terms of the typical values of the magnetic field intensity and of the frequency of emission. Denoting with $\tilde{\nu}$ the physical frequency, i.e. $\tilde{\nu}=$ $\nu / a$, it is clear that $\overrightarrow{\mathcal{B}} / \widetilde{\nu}^{2}=\vec{B} / \nu^{2}$ since the quantities in the numerator and in denominator redshift in the same way. Thus Eq. (3.43) becomes:

$$
\Omega_{\epsilon}=\frac{d \Phi}{d \epsilon}=3.56 \times 10^{-3}\left(\frac{\mathcal{B}}{10^{-9} \mathrm{G}}\right)\left(\frac{100 \mathrm{GHz}}{\tilde{\nu}}\right)^{2}
$$

The typical quantities reported in Eq. (3.45) refer to the present time. It is also conventional to use the same quantities but evaluated at the decoupling epoch. In this case the magnetic field will be of the order of the $\mathrm{mG}$ while the physical frequency (at the decoupling time) will be of the order of $100 \mathrm{THz}$.

\subsection{Faraday effect and radiative transfer equations}

The Faraday rotation rate derived in Eq. (3.43) directly enters the radiative transfer equations. From Eq. (3.39) it is possible to give an order of magnitude estimate of $\Phi$ itself. Indeed, by integrating Eq. (3.39) with respect to $t$, we can obtain that

$$
\Phi=\frac{e^{3}}{2 \pi c m_{\mathrm{e}}^{2} \nu^{2}} \vec{B} \cdot \hat{n} \int x_{\mathrm{e}} n_{0} d t .
$$

Recalling that $a(\tau) d \tau=d t$, the integral appearing in Eq. (3.46) can be easily estimated using Eq. (3.41); the result will be

$$
\int x_{\mathrm{e}} n_{0} d t=\frac{\epsilon\left(\tau, \tau_{0}\right)}{\sigma_{\mathrm{T}}}
$$

For orders of magnitude estimates, one can safely assume that the optical depth, i.e. $\epsilon\left(\tau, \tau_{0}\right)$ is of order unity out to redshift of decoupling, when the polarization is generated [37, 38, 39]. Therefore, after averaging over the polatizations of the magnetic field, the final result can be expressed in terms of $\sqrt{\left\langle\Phi^{2}\right\rangle}$ :

$$
\sqrt{\left\langle\Phi^{2}\right\rangle}=1.6\left(\frac{\mathcal{B}}{10^{-9} \mathrm{G}}\right)\left(\frac{30 \mathrm{GHz}}{\tilde{\nu}}\right)^{2} \mathrm{deg} .
$$

To obtain more accurate estimates of the Faraday rotation rate, the relevant radiative transfer equations must be integrated [37] (see also [40] for an introduction to the radiative transfer equations in CMB physics). As it is well known, the polarization of the CMB is a higher order effect with respect to the temperature anisotropies and it depends crucially upon the properties of the photon phase space distribution that should have a non-vanishing quadrupole moment. Indeed, to zeroth order in the tight-coupling expansion photons and baryons are synchronized so well that the CMB is not polarized. This means, technically, 
that the brightness perturbation associated with the $\mathrm{Q}$ Stokes parameter (i.e. $\Delta_{\mathrm{Q}}$ ) is zero to zeroth order in the tight coupling expansion. Since $\Delta_{\mathrm{Q}}$ measures the degree of linear polarization, the CMB, to this order, is not polarized. However, to first order in the tightcoupling expansion a small amount of linear polarization is generated and it is proportional to the expansion parameter, i.e. $1 / \epsilon^{\prime}$, and to the (zeroth-order) dipole.

This argument disregards the important fact that the $\mathrm{Q}$ and $\mathrm{U}$ Stokes parameters (and their associated brightness fluctuations $\Delta_{\mathrm{Q}}$ and $\Delta_{\mathrm{U}}$ ) are not invariant under rotations. It is possible to take into account this aspect by defining a generalized degree of polarization that is proportional to $\left(\Delta_{\mathrm{U}}^{2}+\Delta_{\mathrm{Q}}^{2}\right)$.

The presence of a magnetic field introduces then further terms in the radiative transfer equations. These terms couple directly the evolution of $\Delta_{\mathrm{Q}}$ to the evolution of $\Delta_{\mathrm{U}}$ and the strength of the coupling is given by the Faraday rotation rate $\omega_{\Phi}$ introduced in Eq. (3.45). Therefore, the relevant radiative transfer equations can be written as ${ }^{7}$

$$
\begin{aligned}
& \Delta_{\mathrm{Q}}^{\prime}+\left(i k \mu+\epsilon^{\prime}\right) \Delta_{\mathrm{Q}}=\frac{\epsilon^{\prime}}{2}\left[1-P_{2}(\mu)\right] S_{\mathrm{Q}}+2 \Omega_{\Phi} \Delta_{\mathrm{U}}, \\
& \Delta_{\mathrm{U}}^{\prime}+\left(i k \mu+\epsilon^{\prime}\right) \Delta_{\mathrm{U}}=-2 \Omega_{\Phi} \Delta_{\mathrm{Q}},
\end{aligned}
$$

where,

$$
S_{\mathrm{Q}}=\Delta_{\mathrm{I} 2}+\Delta_{\mathrm{Q} 0}+\Delta_{\mathrm{Q} 2} .
$$

Eqs. (3.49) and (3.50) are derived by using the following expansions for the brightness perturbations:

$$
\begin{aligned}
& \Delta_{\mathrm{Q}}(\vec{k}, \hat{n}, \tau)=\sum_{\ell}(-i)^{\ell}(2 \ell+1) \Delta_{\mathrm{Q} \ell}(\vec{k}, \tau) P_{\ell}(\hat{k} \cdot \hat{n}), \\
& \Delta_{\mathrm{U}}(\vec{k}, \hat{n}, \tau)=\sum_{\ell}(-i)^{\ell}(2 \ell+1) \Delta_{\mathrm{U} \ell}(\vec{k}, \tau) P_{\ell}(\hat{k} \cdot \hat{n}),
\end{aligned}
$$

where $\vec{k}$ is the momentum of the Fourier expansion, $\hat{k}$ its direction; $\hat{n}$ is the direction of the photon momentum and $\mu=\hat{k} \cdot \hat{n}$. Consequently, in Eq. (3.51), $\Delta_{\mathrm{I} 2}$ denotes the quadrupole moment of the brightness fluctuation associated with the I Stokes parameter, while $\Delta_{\mathrm{Q} 0}$ and $\Delta_{\mathrm{Q} 2}$ denote, respectively, the monopole and quadrupole moment of $\Delta_{\mathrm{Q}}$. For completeness, and to anticipate further considerations on the cross power spectra, we also introduce the expansion for the brightness perturbation associated with the intensity of the radiation field:

$$
\Delta_{\mathrm{I}}(\vec{k}, \hat{n}, \tau)=\sum_{\ell}(-i)^{\ell}(2 \ell+1) \Delta_{\mathrm{I} \ell}(\vec{k}, \tau) P_{\ell}(\hat{k} \cdot \hat{n}) .
$$

An important remark concerns here the symmetry of the radiative transfer equations. In Eqs. (3.49) and (3.50) $\mu$ denotes the projection of the Fourier mode along the direction

\footnotetext{
${ }^{7}$ Here we are only discussing the Faraday effect induced on the polarization in the case of scalar fluctuations of the geometry.
} 
of the photon momentum, i.e. $\mu=\hat{k} \cdot \hat{n}$. In Eqs. (3.49) and (3.50) the magnetic field may introduce an extra preferred direction, so that the evolution equations will depend, ultimately, not only upon $\mu$ but also upon $\hat{B} \cdot \hat{n}$ (see Eq. (3.43) ). Furthermore, the analysis may become even more cumbersome if the magnetic field is allowed not to be uniform (as assumed in the present and in the previous section). To make Eqs. (3.49) and (3.50) more tractable the direction of the magnetic field intensity is taken to lie along the direction of the Fourier mode. Instead of working with $\Delta_{\mathrm{Q}}$ and $\Delta_{\mathrm{U}}$, it is useful to write down Eqs. (3.49) and (3.50) directly in terms of the two associated complex conjugate combinations, i.e.

$$
\mathcal{M}_{ \pm}=\Delta_{\mathrm{Q}} \pm i \Delta_{\mathrm{U}}
$$

Using Eq. (3.55), Eqs. (3.49) and (3.50) give, after some simple algebra,

$$
\mathcal{M}_{ \pm}^{\prime}+\left(i k \mu+\epsilon^{\prime} \pm 2 i \Omega_{\Phi}\right) \mathcal{M}_{ \pm}=\frac{3}{4} \epsilon^{\prime}\left(1-\mu^{2}\right) S_{\mathrm{Q}}
$$

Using the definitions given in Eqs. (3.41) and (3.42), Eq. (3.56) can be integrated easily and the result of the integration is

$$
\mathcal{M}_{ \pm}\left(\vec{k}, \tau_{0}\right)=\frac{3}{4}\left(1-\mu^{2}\right) \int_{0}^{\tau_{0}} \mathcal{K}(\tau) e^{i k \mu\left(\tau-\tau_{0}\right)} e^{\mp \epsilon\left(\tau, \tau_{0}\right) \Omega_{\epsilon}(\hat{B} \cdot \hat{n})} S_{Q}(\tau) d \tau
$$

where $\Omega_{\epsilon}$ is given by Eq. (3.45). This equation can be used for semi-analytical estimates of the mixing between $\Delta_{\mathrm{Q}}$ and $\Delta_{\mathrm{U}}$ to corroborate numerical estimates of the same effect.

The analysis performed in terms of $\Delta_{\mathrm{Q}}$ and $\Delta_{\mathrm{U}}$ can also be expressed in terms of the $\mathrm{E}$ and $\mathrm{B}$ modes. In the latter language, the mixing between $\Delta_{\mathrm{Q}}$ and $\Delta_{\mathrm{U}}$ translates into a mixing between $\mathrm{E}$ and $\mathrm{B}$ modes ${ }^{8}$.

Recalling, in fact, that under clockwise rotations of $\varphi \mathcal{M}_{ \pm}$transform as

$$
\tilde{\mathcal{M}}_{ \pm}=e^{\mp 2 i \varphi} \mathcal{M}_{ \pm}
$$

the combinations

$$
\mathcal{M}_{ \pm}(\hat{n})=\sum_{\ell m} a_{ \pm 2, \ell m} \quad{ }_{ \pm 2} \mathcal{Y}_{\ell m}(\hat{n})
$$

can be expanded in terms of the spin-2 spherical harmonics, i.e. ${ }_{22} \mathcal{Y}_{\ell}^{m}(\hat{n})$, with

$$
a_{ \pm 2, \ell m}=\int d \hat{n} \quad{ }_{ \pm 2} \mathcal{Y}_{\ell m}^{*}(\hat{n})\left(\Delta_{\mathrm{Q}} \pm i \Delta_{\mathrm{U}}\right)(\hat{n}) .
$$

The "electric" and "magnetic" components of polarization are eigenstates of parity and may be defined as

$$
a_{\ell m}^{\mathrm{E}}=-\frac{1}{2}\left(a_{2, \ell m}+a_{-2, \ell m}\right), \quad a_{\ell m}^{\mathrm{B}}=\frac{i}{2}\left(a_{2, \ell m}-a_{-2, \ell m}\right) .
$$

\footnotetext{
${ }^{8}$ It should be clear that the $E$ abd $\mathrm{B}$ modes discussed here are not necessarily related to physical electric and magnetic fields.
} 
These newly defined variables are expanded in terms of ordinary spherical harmonics, $Y_{\ell m}(\hat{n})$

$$
\mathrm{E}(\hat{n})=\sum_{\ell m} a_{\ell m}^{\mathrm{E}} Y_{\ell m}(\hat{n}), \quad \mathrm{B}(\hat{n})=\sum_{\ell m} a_{\ell m}^{\mathrm{B}} Y_{\ell m}(\hat{n})
$$

In connection with the spin-2 spherical harmonics appearing in Eqs. (3.59) and (3.60) it is relevant to mention here that a generic spin-s spherical harmonic is a known concept in the quantum mechanical theory of angular momentum (see, for instance, 41]). A typical quantum mechanical problem is to look for the representations of the operator specifying three-dimensional rotations, i.e. $\hat{R}$; this problem is usually approached within the so-called Wigner matrix elements, i.e. $\mathcal{D}_{m m^{\prime}}^{(j)}(R)=\left\langle j, m^{\prime}|\hat{R}| j, m>\right.$ where $j$ denotes the eigenvalue of $J^{2}$ and $m$ denotes the eigenvalue of $J_{z}$. Now, if we replace $m^{\prime} \rightarrow-\mathrm{s}, j \rightarrow \ell$, we have the definition of spin-s spherical harmonics in terms of the $\mathcal{D}_{-s, m}^{(\ell)}(\alpha, \beta, 0)$, i.e.

$$
\mathcal{s}_{\ell m}(\alpha, \beta)=\sqrt{\frac{2 \ell+1}{4 \pi}} \mathcal{D}_{-\mathrm{s}, m}^{(\ell)}(\alpha, \beta, 0),
$$

where $\alpha, \beta$ and $\gamma$ (set to zero in the above definition) are the Euler angles defined as in [41. If $s=0, \mathcal{D}_{0, m}^{(\ell)}(\alpha, \beta, 0)=\sqrt{(2 \ell+1) / 4 \pi} Y_{\ell m}(\alpha, \beta)$ where $Y_{\ell m}(\alpha, \beta)$ are the ordinary spherical harmonics.

To define properly the cross power spectra we also recall the expansion of the intensity fluctuations of the radiation field (defined in Eq. (3.54)), in terms of spherical harmonics:

$$
\Delta_{\mathrm{I}}(\hat{n})=\sum_{\ell m} a_{\ell m}^{\mathrm{T}} Y_{\ell m}(\hat{n})
$$

where we wrote explicitly $a_{\ell m}^{\mathrm{T}}$ since the fluctuations in the intensity of the radiation field, i.e. $\Delta_{\mathrm{I}}$ are nothing but the fluctuations in the CMB temperature.

Under parity inversion, the components appearing in Eqs. (3.62) and (3.64) transform as

$$
a_{\ell m}^{\mathrm{E}} \rightarrow(-1)^{\ell} a_{\ell m}^{\mathrm{E}}, \quad a_{\ell m}^{\mathrm{B}} \rightarrow(-1)^{\ell+1} a_{\ell m}^{\mathrm{B}}, \quad a_{\ell m}^{\mathrm{T}} \rightarrow(-1)^{\ell} a_{\ell m}^{\mathrm{T}} .
$$

Therefore, the E-modes have the same parity of the T-modes which have, in turn, the same parity of spherical harmonics, i.e. $(-1)^{\ell}$. On the contrary, the B-modes have $(-1)^{\ell+1}$ parity.

The existence of linear polarization allows for 6 different cross power spectra to be determined, in principle, from data that measure the full temperature and polarization anisotropy information. The cross power spectra can be defined in terms of the spectral functions $C_{\ell}^{X, Y}$ where $X$ and $Y$ stand for E, B or T depending on the cross-correlation one is interested in:

$$
C_{\ell}^{X, Y}=\frac{1}{2 \pi^{2}} \int k^{2} d k \sum_{m=-\ell}^{\ell} \frac{\left(a_{\ell m}^{X}\right)^{*} a_{\ell m}^{Y}}{(2 \ell+1)} .
$$


Therefore, if we are interested in the TE correlations we just have to set $X=\mathrm{T}$ and $Y=\mathrm{E}$ and use the relevant expansions given above. In the following, we will denote the correlations as TT, EE, BB, TB and so on. This notation refers to the definition given in Eq. (3.66).

Let us now see how the definition (3.66) works. Suppose we are interested in the TT correlations, i.e. the usual and well known temperature correlations. From Eq. (3.66) we will have

$$
C_{\ell}^{\mathrm{TT}}=\frac{1}{2 \pi^{2}} \int k^{2} d k \sum_{m=-\ell}^{\ell} \frac{\left[a_{\ell m}^{\mathrm{T}}(k)\right]^{*} a_{\ell m}^{\mathrm{T}}(k)}{(2 \ell+1)} .
$$

Now, from Eq. (3.64), using the orthogonality of spherical harmonics, we have that

$$
a_{\ell m}^{\mathrm{T}}(k)=\int d \hat{n} Y_{\ell m}(\hat{n}) \Delta_{\mathrm{I}}(\vec{k}, \hat{n})
$$

Inserting Eq. (3.68) into Eq. (3.67) and recalling the expansion of $\Delta_{\mathrm{I}}(\vec{k}, \hat{n})$ in terms of Legendre polynomials we get

$$
C_{\ell}^{\mathrm{TT}}=\frac{2}{\pi} \int d k k^{2}\left|\Delta_{\mathrm{I} \ell}\right|^{2}
$$

To get to Eq. (3.69) the following two identities have been used, i.e.

$$
\begin{aligned}
& \int d \hat{n} P_{\ell^{\prime}}(\hat{k} \cdot \hat{n}) Y_{\ell m}^{*}(\hat{n})=\frac{4 \pi}{(2 \ell+1)} \delta_{\ell \ell^{\prime}}, \\
& \sum_{m=-\ell}^{\ell} Y_{\ell m}^{*}(\hat{k}) Y_{\ell m}(\hat{n})=\frac{2 \ell+1}{4 \pi} P_{\ell}(\hat{k} \cdot \hat{n}) .
\end{aligned}
$$

The second identity in Eq. (3.70) has been already exploited in Eq. (2.17).

In similar ways, different expressions for the other correlations may be obtained. Notice that Eq. (3.69) is a consequence of the specific conventions adopted in Eq. (3.54). In particular note that the a factor $(2 \ell+1)$ is included in the expansion. It must be clearly said that this is matter of conventions. For instance, in Refs. [40] and [42] the factor $(2 \ell+1)$ as well as the factor $(-i)^{\ell}$ are included in the expansion ${ }^{9}$. On the contrary, in Ref. [43, the authors did not include the factor $(2 \ell+1)$ in the expansions for the brightness perturbations that we defined in Eqs. (3.52), (3.53) and (3.54). Consequently, the cross correlations will inherit extra-terms that are simply a consequence of the different conventions adopted. So, for instance, in Eq. (3.69), and in the conventions of [43, a factor $(2 \ell+1)^{2}$ typically appears in the denominator.

\footnotetext{
${ }^{9}$ Notice that the authors of Ref. [39] do not include the factor $(-i)^{\ell}$ but do include the factor $(2 \ell+1)$ in the expansion of the various brightness perturbations.
} 
Recalling the connection between the Wigner matrix elements and the spin-s spherical harmonics, i.e. Eq. (3.63), it is possible to show that, under complex conjugation,

$$
a_{ \pm 2, \ell m}^{*}=(-1)^{m} a_{\mp 2, \ell m}, \quad\left(a_{\ell, m}^{\mathrm{T}, \mathrm{E}, \mathrm{B}}\right)^{*}=(-1)^{m} a_{\ell,-m}^{\mathrm{T}, \mathrm{E}, \mathrm{B}},
$$

where the second equality follows from the first one by using Eq. (3.61). It is then possible to show that while the TB and EB correlators are parity-odd, all the other correlators (i.e. TT, BB, EE, TE) are parity even.

\subsection{Limits on uniform magnetic fields from Faraday effect}

In the absence of magnetic fields, the scalar fluctuations of the geometry only generate E-modes. The presence of a magnetic field induces, however, a mixing between E-modes and B-modes. Consequently, if we have an initial E-mode generated by scalar metric perturbations, the presence of a magnetic field induces a non-vanishing B-mode. In the case of scalar fluctuations, a non-vanishing TE power spectrum arises directly as a result of the CMB polarization as it can be verified by studying the first-order tight coupling expansion. Recently, the WMAP collaboration [44, 45] measured the TE correlations. As discussed in [46], the induced TB angular power spectrum is, roughly $\Omega_{\epsilon}$ times smaller than the initial $T E$ correlations. From the WMAP measurement, the measured value of the $T E$ correlation and the absence of the TB correlations allows to set a limit on $\Omega_{\epsilon}$. At a given frequency, the limit on $\Omega_{\epsilon}$ can be translated into a bound on the magnetic field intensity, i.e.

$$
\mathcal{B}_{0}<10^{-8} \mathrm{G}
$$

at a typical (present) frequency of $30 \mathrm{GHz}$ [4]. The values of the TE and EE correlations induced by the presence of a magnetic field are $\Omega_{\epsilon}^{2}$ times smaller than their values in the absence of the magnetic fields. In Fig. 4 the autocorretaion of the $B$-modes of CMB polarization is reported in the case of a uniform magnetic field (recall that $\Omega_{\epsilon}$ is defined in Eq. (3.45)). Notice that when $2 \Omega_{\epsilon} \simeq 1$ the $T E$ (as well as the the other correlations) are strongly damped and the differential Faraday rotation has a net depolarizing effect 39].

We conclude this section by remarking that Faraday rotation is not the only way to generate $B$-modes. In fact:

- B-modes can be generated directly by fully inhomogeneous magnetic fields (see following sections);

- B-modes can be generated by gravitational waves (in Fig. 4) the GW contribution is reported for a value of the tensor-to scalar ratio compatible with the present WMAP data); 


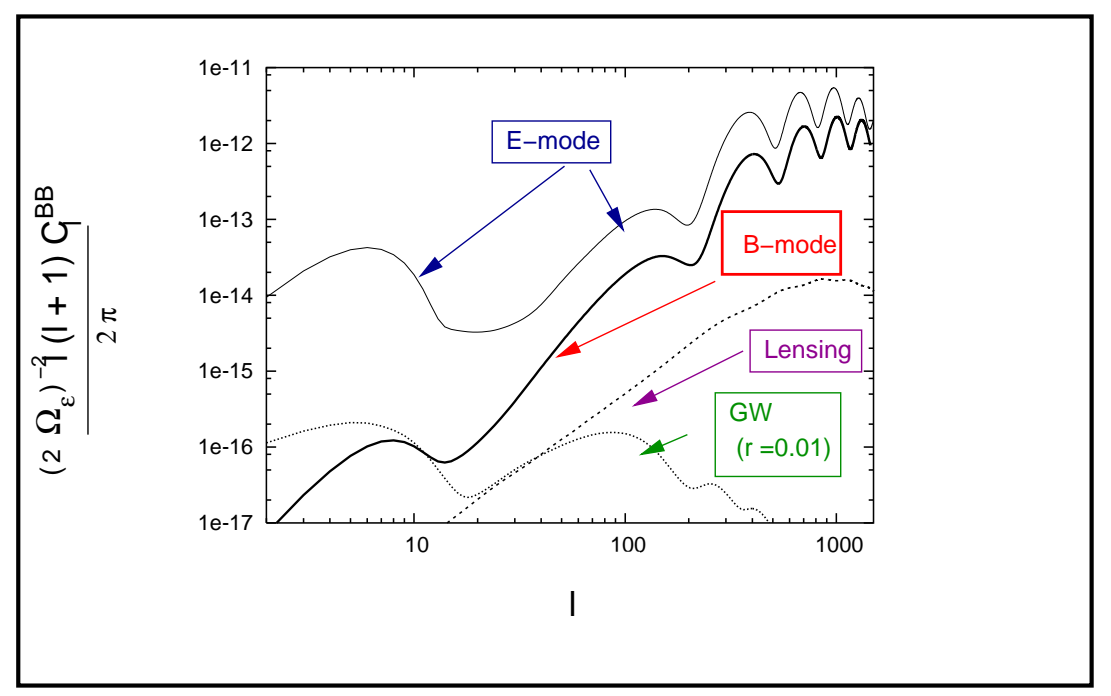

Figure 4: Angular autocorrelation of B-modes of $\mathrm{CMB}$ polarization generated by a uniform magnetic field in a cosmic concordance model (adapted from Ref. [46]). Notice that the vertical axis of has been rescaled by $\left(2 \Omega_{\epsilon}\right)^{-2}$ (see Eq. (3.45) ). With the dashed line marked with "lensing" the possible B-mode arising from weak lensing is reported. The B-mode induced by GW is also reported (dotted line) for a typical value of the tensor to scalar ratio $r=0.01$. 
- B-modes can be generated by weak lensing.

To this list one should also add the possibility that some other form of birefringence is allowed (as elaborated in the derivation of Eq. (3.34)). In connection with the GW contribution, it should be noted that $r$ (denoting the ration between the tensor and scalar (primordial) power spectra) is limited from above, by current experimental data, i.e. $r<0.53$. In Fig. 4 the contribution of the B-mode induced by GW is illustrated in the case $r=0.01$.

The peculiar feature of the Faraday rotation signal induced by a uniform magnetic field is the frequency dependence. In fact, by going at higher frequencies, the signal gets reduced as $\nu^{-2}$. On the contrary the B-modes induced by gravitational waves does not depend upon the frequency. The birefringence induced by a dynamical quintessence (pseudo-scalar) field is also independent on the frequency. The need of multifrequency determination of Faraday rotation is then clear and this point has been also correctly emphasized in [47, 48]. In [4] a scheme for the treatment of Faraday rotation in the case of fully inhomogeneous magnetic fields has been studied and along a similar line another analysis has also been presented in [49].

In the following years on the CMB side the Planck mission [50] will give us, hopefully, important clues concerning CMB polarization. For the purposes of the present subject it is appropriate to recall the Planck will operate in 9 frequency channels ranging from 30 to 857 GHz. Multifrequency Faraday rotation measurements may become possible. Unfortunately, owing to the fact that the nominal Planck sensitivity to polarization is larger at higher frequencies, these measurements may also be difficult. In fact, Faraday rotation signals are suppressed by the square of the frequency and are therefore smaller at high frequencies. Other instruments dedicated to the analysis of CMB polarization may also give important clues. These experiments include polarization-sensitive balloon borne experiments (like the polarization-sensitive version of Boomerang [51, 52] and its possible improvements) as well as ground based arrays like the promising QUIET (Q/UImagingExperimenT) [53]. On the radio-astronomical side, the very ambitious SKA (Square Kilometer Array) project will be able to give us precious full-sky surveys of Faraday rotation measurements [54] (see also [55, [56]). The SKA experiment should cover a frequency range between 0.1 and $20 \mathrm{GHz}$.

\section{One-fluid plasma description}

Since a fully inhomogeneous magnetic field acts as a source term in the evolution equations of the gravitational perturbations, the $\mathrm{CMB}$ observables may be affected. To investigate this situation, the relevant system of equations describing the large-scale magnetic fields is slightly different from the one introduced in Eqs. (3.12), (3.13) and (3.14) (where, however, 
the displacement current may be neglected). In fact, we are here interested in the dynamics taking place for typical frequencies much smaller than $\omega_{\mathrm{p}, \mathrm{e}}$ and $\omega_{\mathrm{p}, \mathrm{i}}$. In this situation, from the two-fluid equations of Eqs. (3.12), (3.13) and (3.14) it is possible to derive various one-fluid plasma descriptions by defining a set of appropriate one-fluid variables such like the total current [24, 57]

$$
\vec{J}=e\left(n_{i} \vec{v}_{\mathrm{i}}-n_{\mathrm{e}} \vec{v}_{\mathrm{e}}\right)
$$

the center-of-mass velocity

$$
\vec{v}=\frac{m_{\mathrm{i}} \vec{v}_{\mathrm{i}}+m_{\mathrm{e}} \vec{v}_{\mathrm{e}}}{m_{\mathrm{e}}+m_{\mathrm{i}}}
$$

and so on. Among the one-fluid descriptions, a particularly simple possibility is provided by magnetohydrodynamics (MHD) that can be characterized by the following set of conditions:

$$
\vec{\nabla} \cdot \vec{E}=0, \quad \vec{\nabla} \cdot \vec{B}=0, \quad \vec{\nabla} \cdot \vec{J}=0 .
$$

As mentioned above, the derivative of the electric field is neglected in MHD analog of Eq. (3.14). Moreover, since the current is solenoidal, as the magnetic field intensity, it follows, from Maxwell's equations, that

$$
\vec{J}=\frac{1}{4 \pi} \vec{\nabla} \times \vec{B} .
$$

The induced (Ohmic) electric field is therefore given, in the absence of Hall and thermoelectric terms, by

$$
\vec{E}+\vec{v} \times \vec{B}=\frac{1}{4 \pi \sigma} \vec{\nabla} \times \vec{B},
$$

where $\sigma$ is the conductivity and where we used units where the speed of light is equal to 1 . In a relativistic plasma $\sigma \simeq T / \alpha_{\mathrm{em}}$ while in a non-relativistic plasma $\sigma \simeq\left(T / \alpha_{\mathrm{em}}\right)\left(T / m_{\mathrm{e}}\right)^{1 / 2}$. Since the conductivity increases as we go back in time, in the early Universe the condition $\vec{E}+\vec{v} \times \vec{B} / c \simeq 0$ is approximately verified. Under these conditions, two (approximate) conservations laws may be derived, namely the magnetic flux conservation

$$
\frac{d}{d \tau} \int_{\Sigma} \vec{B} \cdot d \vec{\Sigma}=-\frac{1}{4 \pi \sigma} \int_{\Sigma} \vec{\nabla} \times \vec{\nabla} \times \vec{B} \cdot d \vec{\Sigma}
$$

and the magnetic helicity conservation

$$
\frac{d}{d \tau}\left(\int_{V} d^{3} x \vec{A} \cdot \vec{B}\right)=-\frac{1}{4 \pi \sigma} \int_{V} d^{3} x \vec{B} \cdot \vec{\nabla} \times \vec{B}
$$

In Eq. (4.6) $\Sigma$ is an arbitrary closed surface that moves with the plasma. According to Eq. (4.6), in MHD the magnetic field has to be always solenoidal (i.e. $\vec{\nabla} \cdot \vec{B}=0$ ). Thus, the magnetic flux conservation implies that, in the superconducting limit (i.e. $\sigma \rightarrow \infty$ ) the magnetic flux lines, closed because of the transverse nature of the field, evolve always glued together with the plasma element. In this approximation, as far as the magnetic field evolution is concerned, the plasma is a collection of (closed) flux tubes. The theorem of 
flux conservation states then that the energetical properties of large-scale magnetic fields are conserved throughout the plasma evolution.

While the flux conservation concerns the energetic properties of the magnetic flux lines, the magnetic helicity, i.e. Eq. (4.7), concerns chiefly the topological properties of the magnetic flux lines. In the simplest situation, the magnetic flux lines will be closed loops evolving independently in the plasma and the helicity will vanish. There could be, however, more complicated topological situations [102] where a single magnetic loop is twisted (like some kind of Möbius stripe) or the case where the magnetic loops are connected like the rings of a chain: now the non-vanishing magnetic helicity measures, essentially, the number of links and twists in the magnetic flux lines [24]. Furthermore, in the superconducting limit, the helicity will not change throughout the time evolution. The conservation of the magnetic flux and of the magnetic helicity is a consequence of the fact that, in ideal MHD, the Ohmic electric field is always orthogonal both to the bulk velocity field and to the magnetic field. In the resistive MHD approximation this conclusion may not apply 10

The quantity at the right-hand-side of Eq. (4.7), i.e. $\vec{B} \cdot \vec{\nabla} \times \vec{B}$ is called magnetic gyrotropy and it is a gauge-invariant measure of the number of contact points in the magnetic flux lines. The magnetic helicity (or the magnetic gyrotropy) and the Lorentz force can be used to give a general classification of the possible inhomogeneous magnetic field configurations that are relevant in a MHD context. Since the current density is solenoidal, we have that, in MHD,

$$
\vec{F}_{\mathrm{B}}=\vec{J} \times \vec{B} \simeq \frac{1}{4 \pi}(\vec{\nabla} \times \vec{B}) \times \vec{B} .
$$

Looking at the expressions of the magnetic gyrotropy and of the Lorentz force, there are, in principle, two extreme situations that may arise:

- in the first case $\vec{\nabla} \times \vec{B} \times \vec{B}=0$ and the magnetic gyrotropy, i.e. $\vec{B} \cdot \vec{\nabla} \times \vec{B}$, is maximal;

- in the second case the Lorentz force is maximal and the magnetic gyrotropy vanishes.

In the case when the Lorentz force term vanishes, the configuration is said to be forcefree. In this limit the MHD equations (coupled with the Navier-Stokes equation) become particularly simple and appropriate for the deduction of useful scaling laws [24, 59, 60, 61. Fully inhomogeneous magnetic field configurations may then gravitate and affect the evolution equations of the tensor, vector and scalar modes of the geometry. Therefore, in the following section, the evolution equations of metric fluctuations will be investigated in the

\footnotetext{
${ }^{10}$ The presence (or absence) of magnetic helicity plays an important rôle for the possibility of inverse cascades in the pre-recombination plasma. In particular, one of the evolutions of the correlation scale presented in Fig. 1 refers to the case where the magnetic field is helical [58].
} 
presence of fully inhomogeneous magnetic fields. MHD equations in curved space-times are also relevant for a number of different problems arising in the physics of large-scale magnetic field. Concerning the various aspects of this subject see, for instance, Refs. 62, 63, 64 and references therein.

As a last comment, we would like to stress that the magnetic helicity as well as the magnetic gyrotropy are parity-odd quantities. Therefore, recalling the considerations related to Eq. (3.71), if sources of gyrotropy and helicity are present after equality the BE and TB correlators may not be zero. This point has been discussed in [65] (see, however, also [47]).

\subsection{Fully inhomogeneous magnetic correlators}

Nearly all mechanisms able to generate large scale magnetic fields imply the existence of a stochastic background of magnetic disturbances that could be written, in Fourier space, as 11

$$
\left\langle B_{i}(\vec{k}, \tau) B_{j}^{*}(\vec{p}, \tau)\right\rangle=(2 \pi)^{3} P_{i j}(k) \delta^{(3)}(\vec{k}-\vec{p}),
$$

where

$$
P_{i j}(k)=\mathcal{Q}(k)\left(\delta_{i j}-\frac{k_{i} k_{j}}{k^{2}}\right), \quad \mathcal{Q}(k)=\mathcal{Q}_{0} k^{m} .
$$

From Eq. (4.10) the magnetic field configuration of Eq. (4.9) depends on the amplitude of the field $\mathcal{Q}_{0}$ and on the spectral index $m$.

It is easy to check that, in the case of the configuration (4.9) the magnetic gyrotropy is vanishing, i.e.

$$
\langle\vec{B} \cdot \vec{\nabla} \times \vec{B}\rangle=0, \quad\left\langle(\vec{B} \cdot \vec{\nabla} \times \vec{B})^{2}\right\rangle=0,
$$

where the second expression denotes, for short, the two-point function of the magnetic gyrotropy. On the contrary it should also be clear that, from Eq. (4.9), that $\langle(\vec{\nabla} \cdot[\vec{\nabla} \times$ $\vec{B}) \times \vec{B}]\rangle \neq 0$.

There are situations where magnetic fields are produced in a state with non-vanishing gyrotropy (or helicity) 66, 67, 65, 68. In this case, the two point function can be written in the same form given in Eq. (4.9)

$$
\left\langle B_{i}(\vec{k}, \tau) B_{j}^{*}(\vec{p}, \tau)\right\rangle=(2 \pi)^{3} \tilde{P}_{i j}(k) \delta^{(3)}(\vec{k}-\vec{p}),
$$

but where now

$$
\tilde{P}_{i j}(k)=\mathcal{Q}(k)\left(\delta_{i j}-\frac{k_{i} k_{j}}{k^{2}}\right)+i \tilde{\mathcal{Q}}(k) \epsilon_{i j \ell} \frac{k^{\ell}}{k}, \quad \tilde{\mathcal{Q}}(k)=\tilde{\mathcal{Q}}_{0} k^{\tilde{m}} .
$$

\footnotetext{
${ }^{11}$ For the Fourier transforms we use the following conventions: $B_{i}(\vec{x})=(2 \pi)^{-3} \int d^{3} k e^{-i \vec{k} \cdot \vec{x}} B_{i}(\vec{k})$ and, conversely, $B_{i}(\vec{k})=\int d^{3} x e^{i \vec{k} \cdot \vec{x}} B_{i}(\vec{x})$.
} 
From Eq. (4.13) we can appreciate that, on top of the parity-invariant contribution (already defined in Eqs. (4.9) and (4.10), there is a second term proportional to the Levi-Civita $\epsilon_{i j \ell}$. In Fourier space, the introduction of gyrotropic configurations implies also the presence of a second function of the momentum $\tilde{\mathcal{Q}}(k)$.

As it will become more plausible from the considerations reported in Section 5, the two-point function determines, eventually, the higher-order correlation functions. Since the contribution of fully inhomogeneous magnetic fields to the energy-momentum tensor is quadratic, it is rather frequent, in various calculations, to evaluate four-point correlation functions. Fourier space is not always the best framework for the evaluation of higher-order correlation functions. Indeed, a swifter derivation of various results can be achieved, in some cases, by staying in real space (and by eventually Fourier transforming the final result). Let us therefore elaborate on the real space correlators associated with the configurations discussed in Eqs. (4.9) and (4.12).

The real space two-point function can be written, for instance, as

$$
\mathcal{G}_{i j}(r)=\left\langle B_{i}(\vec{x}+\vec{r}) B_{j}(\vec{x})\right\rangle=\mathcal{F}_{1}(r) \delta_{i j}+\mathcal{F}_{2}(r) r_{i} r_{j}+\mathcal{F}_{3}(r) \epsilon_{i j k} r^{k} .
$$

If $\mathcal{F}_{3}(r)=0$ then we are in the situation described by Eq. (4.9). If $\mathcal{F}_{3}(r) \neq 0$ the framework is the one defined by Eq. (4.12).

Consider, for instance, the case $\mathcal{F}_{3}(r)=0$. Then, using the parametrization (4.14), Eqs. (4.9) and (4.10) lead to

$$
\mathcal{G}_{i j}(r)=\mathcal{G}_{\perp}(r) \delta_{i j}+\left[\mathcal{G}_{\|}(r)-\mathcal{G}_{\perp}(r)\right] \frac{r_{i} r_{j}}{r^{2}}
$$

where

$$
\begin{aligned}
& \mathcal{G}_{\perp}(r)=\frac{4}{(2 \pi)^{2}} \int k^{2} d k \mathcal{Q}(k)\left[j_{0}(k r)-\frac{j_{1}(k r)}{k r}\right], \\
& \mathcal{G}_{\|}(r)=\frac{8}{(2 \pi)^{2}} \int k^{2} d k \mathcal{Q}(k) \frac{j_{1}(k r)}{k r}
\end{aligned}
$$

where $j_{0}(k r)$ and $j_{1}(k r)$ are the usual spherical Bessel functions 69, 170]:

$$
j_{0}(k r)=\frac{\sin k r}{k r}, \quad j_{1}(k r)=\frac{\sin k r}{(k r)^{2}}-\frac{\cos k r}{k r} .
$$

Another useful parametrization of the real space correlators is the one where the functions $F_{1}(r)$ and $F_{2}(r)$ are written as derivatives of a function $f\left(r^{2}\right)$ whose asymptotic behaviour can be determined from the trace of the two-point function [66. More precisely, in the case $F_{3}(r)=0$, the functions appearing in Eq. (4.14) can be writtten as

$$
\mathcal{F}_{1}(r)=\frac{\partial}{\partial r^{2}}\left[r^{2} f\left(r^{2}\right)\right], \quad \mathcal{F}_{2}(r)=-\frac{\partial}{\partial r^{2}}\left[f\left(r^{2}\right)\right] .
$$


With the definitions (4.18), Eq. (4.14) is clearly transverse (i. e. $\partial^{i} \mathcal{G}_{i j}=0$ ). Moreover, defining as $\mathcal{G}(r)=\operatorname{Tr}\left[\mathcal{G}_{i j}\right]$, we also have

$$
r \mathcal{G}(r)=2 \frac{\partial}{\partial r^{2}}\left[r^{3} f\left(r^{2}\right)\right]
$$

It is often useful, in practical estimates, to regularize the two-point function by using an appropriate "windowing" 71]. Two popular windows are, respectively, the Gaussian and the top-hat functions, i.e.

$$
\mathcal{W}_{\mathrm{g}}(k, L)=e^{-\frac{k^{2} L^{2}}{2}}, \quad \mathcal{W}_{\mathrm{th}}(k, L)=\frac{3}{k L} j_{1}(k L) .
$$

For instance, the regularized trace of $\mathcal{G}_{i j}(r)$ with Gaussian filter can be obtained from the previous expressions by shifting $\mathcal{Q}(k) \rightarrow \mathcal{Q}(k) W_{\mathrm{g}}(k, L)$. The result is

$$
\mathcal{G}(r)=\frac{2 \mathcal{Q}_{0}}{(2 \pi)^{2}} \frac{1}{L^{3+m}} F\left(\frac{m+3}{2}, \frac{3}{2},-\frac{r^{2}}{4 L^{2}}\right),
$$

where $F(a, b, x) \equiv{ }_{1} F_{1}(a, b, x)$ is the confluent hypergeometric function [69, 70]. Notice that the integral appearing in the trace converges for $m>-3$. The amplitude of the magnetic power spectrum $\mathcal{Q}_{0}$ can be traded for $\mathcal{G}(0) \equiv B_{L}^{2}$ (as suggested, for instance, in [72]). From Eq. (4.21) we have that $\mathcal{Q}_{0}$ becomes

$$
\mathcal{Q}_{0}=\frac{(2 \pi)^{m+5}}{2} \frac{k_{L}^{-(3+m)}}{\Gamma\left(\frac{m+3}{2}\right)} B_{L}^{2},
$$

where $k_{L}=2 \pi / L$.

In the real space approach the higher order correlators are reduced to the calculation of derivatives of special functions (depending upon the regularization scheme). For instance, we can compute easily, in general terms the correlation function for the energy density fluctuations. Defining

$$
\delta \rho_{B}(\vec{x})=\frac{1}{8 \pi} B_{i}(\vec{x}) B^{i}(\vec{x})
$$

it is sometimes important to evaluate

$$
\mathcal{E}(r)=\left\langle\delta \rho_{B}(\vec{x}) \delta \rho_{B}(\vec{y})\right\rangle-\left\langle\delta \rho_{\mathrm{B}}(\vec{x})\right\rangle\left\langle\delta \rho_{B}(\vec{y})\right\rangle
$$

By using the decomposition (4.14) we will have (taking for simplicity the case $F_{3}(r)=0$ ),

$$
\begin{aligned}
\mathcal{E}(r) & =\frac{1}{32 \pi^{2}}\left[3 F_{1}^{2}(r)+2 r^{2} F_{1}(r) F_{2}(r)+r^{4} F_{2}^{2}(r)\right. \\
& =\frac{1}{32 \pi^{2}}\left[3 f^{2}\left(r^{2}\right)+4 r^{2} f\left(r^{2}\right) \frac{\partial f}{\partial r^{2}}+2 r^{4}\left(\frac{\partial f}{\partial r^{2}}\right)^{2}\right]
\end{aligned}
$$


where the second equality follows from Eq. (4.18).

With these (or similar techiques) higher order correlation functions for magnetic inhomogeneities may be computed for the interesting observables like the Lorentz force [72], the magnetic gyrotropy [66] and so on.

It is appropriate to comment here on the parametrizations usually employed in the analysis of the various mechanisms for the magnetic field production in the early Universe. This topic (together with other related subjects) has been reviewed in Ref. [63], and, consequently that discussion will not be repeated here. For sake of simplicity consider the mechanisms relying on super-adiabatic amplification of vacuum fluctuations of the gauge fields (see also Fig. 2). In this context what one is led to compute is the amplification of the magnetic field from some initial (vacuum) state. The precise amount of amplification (as well as the spectral properties of the final configuration) is determined by the features of the pump field that changes from model to model. Therefore, initially (i.e. for $\tau \rightarrow-\infty$ ) the quantum mechanical operator related to the magnetic field intensity can be decomposed as

$$
\hat{B}_{i}^{\mathrm{in}}(\vec{x}, \tau)=\frac{i \epsilon_{m n i}}{(2 \pi)^{3}} \sum_{\alpha} e_{n}^{\alpha} \int d^{3} k k_{m}\left[\hat{a}_{\vec{k}, \alpha} f_{k}(\tau) e^{-i \vec{k} \cdot \vec{x}}+\hat{a}_{\vec{k}, \alpha}^{\dagger} f_{k}^{*}(\tau) e^{i \vec{k} \cdot \vec{x}}\right]
$$

where, within the present conventions, $\left[\hat{a}_{\vec{k}, \alpha}, \hat{a}_{\vec{p}, \beta}^{\dagger}\right]=(2 \pi)^{3} \delta_{\alpha \beta} \delta^{(3)}(\vec{k}-\vec{p})$. For $\tau \rightarrow+\infty$

$$
\hat{B}_{\ell}^{\text {out }}(\vec{x}, \tau)=\frac{i \epsilon_{a b \ell}}{(2 \pi)^{3}} \sum_{\beta} e_{b}^{\beta} \int d^{3} k k_{a}\left[\hat{b}_{\vec{k}, \beta} g_{k}(\tau) e^{-i \vec{k} \cdot \vec{x}}+\hat{b}_{\vec{k}, \beta}^{\dagger} g_{k}^{*}(\tau) e^{i \vec{k} \cdot \vec{x}}\right] .
$$

Since both sets of modes are complete, the old modes can be expressed in terms of the new ones, i.e.

$$
f_{k}(\tau)=c_{+}(k) g_{k}(\tau)+c_{-}(k) g_{k}^{*}(\tau) .
$$

Notice that $f_{k}(\tau)$ are normalized, as $\tau \rightarrow-\infty$ as $e^{-i k \tau} / \sqrt{2 k}$, since we demand that the initial state is the vacuum. Therefore, with this normalization, $c_{-}(k)$, for $\tau \rightarrow+\infty$ parametrizes the relevant mode-mixing.

Inserting Eq. (4.28) back into Eq. (4.26) and imposing the continuity of the operators we also have

$$
\begin{aligned}
& \hat{b}_{\vec{k}, \alpha}=c_{+}(k) \hat{a}_{\vec{k}, \alpha}+c_{-}(k)^{*} \hat{a}_{\vec{k}, \alpha}^{\dagger}, \\
& \left|c_{+}(k)\right|^{2}-\left|c_{-}(k)\right|^{2}=1 .
\end{aligned}
$$

The final value of the two-point function can then be obtained as

$$
\langle 0| \hat{B}_{i}(\vec{x}+\vec{r}) \hat{B}_{j}\left(\vec{x}|0\rangle \simeq \frac{1}{(2 \pi)^{3}} \int d^{3} k\left(\delta_{i j}-\frac{k_{i} k_{j}}{k^{2}}\right) \mathcal{Q}(k) e^{-i \vec{k} \cdot \vec{r}}\right.
$$


where now $\mathcal{Q}(k) \simeq k\left|c_{-}(k)\right|^{2}$. In deriving Eq. (4.31) we used the identity

$$
\left|g_{k}(\tau)\right|^{2}=\left(1+2\left|c_{-}(k)\right|^{2}\right)\left|f_{k}(\tau)\right|^{2}-c_{-}(k) c_{+}(k) f_{k}^{*}(\tau)^{2}-c_{-}^{*}(k) c_{+}^{*}(k) f_{k}(\tau)^{2},
$$

together with Eq. (4.30). Notice that the the second and third terms in Eq. (4.32) are typically oscillating and they have been dropped in Eq. (4.31).

Super-adiabatic amplification alone leads always to correlation functions falling in the general class introduced in Eq. (4.14) but with $\mathcal{F}_{3}(r)=0$. The primordial spectrum must be processed and the finite values of the thermal diffusivity as well as of the conductivity lead to an effective ultraviolet cut-off 63 .

\subsection{Primordial or astrophysical seeds?}

We are now are going to give some examples of magnetic fields that may be generated in the early stages of the life of the Universe. This topic has been already discussed in different review articles (see, for instance, for a short account [48] and 63, for a more extended review). As elaborated in the introduction, the mechanisms for the generation of magnetic fields that may be potentially relevant for CMB physics can be divided, broadly speaking into two categories: mechanisms operating inside the Hubble radius (like phase transitions occurring, for instance, at the electroweak or at the QCD epoch) and mechanisms where the correlation scale of the field grows larger than the Hubble radius (as in the case of inflationary mechanisms). Furthermore, there is also the appealing possibility of so-called mixed mechanisms where the magnetic field is created, initially, in the context of some (conventional or unconventional) inflationary model. Then the various modes of the primordial spectrum of the hypercharge field will reenter at different times during the radiation epoch and, at this stage, will be further amplified by the processes taking place inside the Hubble radius.

In the case of the electroweak phase transition (see, for instance, 62 for a comprehensive review on this subject) the typical strength of the produced seeds may change depending on the order of the phase transition (PT). We do know that, in the framework of the minimal standard model of particle interaction the phase transition cannot be strongly first-order for values of the Higgs mass larger than the mass of the $\mathrm{W}$ boson. This conclusion was reached by lattice studies of the phase diagram of the electroweak phase transition [73]. Let us now consider, separately, the cases when the PT is strongly first-order and second order.

In a first-order phase transition the phases of the complex order parameter of the nucleated bubbles are not correlated. The bubbles of the broken phase expand at a velocity $v \sim 10^{-3} c$ where $c$ is the speed of light. At the epoch of the electroweak phase transition the temperature of the plasma is of the order of $100 \mathrm{GeV}$ while the Hubble radius, 
i.e. $H_{\text {ew }}^{-1} \sim 3 \mathrm{~cm}$. The average distance between the centers of the nucleated bubbles is of the order of $10^{-8} H_{\text {ew }}^{-1}[62,75]$. The finite conductivity effects will give rise to diffusion whose consequence will be to smooth out the oscillations of the created magnetic field. The magnetic flux will the escape the intersection regions and penetrate the colliding bubbles where the evolution is governed by ordinary MHD. The magnetic fields will typically have a correlation scale much smaller than the Hubble radius. Even granting for a rather efficient diffusion of the magnetic flux, the correlation scale of the magnetic field will be at, at most, as large as the Hubble radius. In the absence of any form of inverse cascade we will have that the typical correlation scale at the onset of galactic rotation will be of the order of 10 to 100 A.U. (recall 1 A.U. $=1.49 \times 10^{13} \mathrm{~cm}$ ). The conservative estimate of Ref. [76] is even smaller, i.e. the authors get a magnetic field $|\vec{B}| \sim 10^{-10} \mathrm{G}$ over a typical correlation scale of 0.1 A. U. . As suggested in the introduction, it is plausible that some form of inverse cascade may eventually take place between the electroweak epoch and the time of electron positron annihilation [58] (see also [63] and references therein). If the PT is of second order the situation is even worse. The correlation scale is likely to be of the order of $10 / T_{\text {ew }}$, i.e. $10^{-16} H_{\text {ew }}^{-1}$ at $T_{\text {ew }}$.

There have been also ideas concerning a possible generation of magnetic fields at the time of the QCD phase transition occurring roughly at $T \sim 140 \mathrm{MeV}$, i.e. at the moment when free quarks combine to form colorless hadrons. At this time the Hubble radius is of the order of $4 \times 10^{4} \mathrm{~m}$. The mechanism here is always related to the idea of a Biermann battery with thermoelectric currents developed at the QCD time ${ }^{12}$. Since the strange quark is heavier than the up and down quarks there may be the possibility that the quarks develop a net positive charge which is compensated by the electric charge in the leptonic sector. Again, invoking the dynamics of a first-order phase transition, it is argued that the shocks affect leptons and quarks in a different way so that electric currents are developed as the bubble wall moves in the quark-gluon plasma. In [77] the magnetic field has been estimated to be $|\vec{B}| \sim \mathrm{G}$ at the time of the QCD phase transition and with typical scale of the order of the meter at the same epoch.

In [78, 79] it has been pointed out that, probably, the magnetic fields generated at the time of QCD phase transition may be much stronger than the ones estimated in [77]. The authors of [78, 79] argue that strong magnetic fields may be generated when the broken and symmetric phase of the theory coexist. The magnetic fields generated at the boundaries between quark and hadron phases can be, according to the authors, as large as $10^{6} \mathrm{G}$ over scales of the order of the meter (and possibly even larger) at the time of the QCD phase transition. At the onset of gravitational collapse and over a typical scale of the Mpc the

\footnotetext{
${ }^{12}$ See later in this Section for a general discussion of "batteries" in the framework of the generalized Ohm law
} 
magnetic field will be of the order of $10^{-26} \mathrm{G}$ with white noise spectrum ( $m \sim 0$ in the notations of this section) [78.

A model of variation of gauge couplings motivated by the evolution of the internal dimensions 81] leads to a variety of spectra that may be different depending on the number of extra-dimensions and on their rate of evolution (see also [82, 83, 84]). While a phase of expanding internal dimensions may lead to large magnetic fields, the allowed region in the parameter space gets reduced in the case of contracting extra-dimensions. In [81] only the gauge zero-modes have been considered. In a recent calculation [85], the rôle of the internal momenta has been taken into account with the result that also in the contracting case large magnetic seeds may be produced. In the case of expanding internal dimensions the spectral index is $m \sim-1-n \mu$ where $n$ is the number of internal dimensions and $\mu$ is the exponent parametrizing, on conformal time, their evolution. In the case of Kasner models $81 \mu=\sqrt{3 n /(n+2)}$. If internal dimensions contract we have, effectively, $m \sim 0$ 85 .

Large-scale magnetic fields can also be produced in the context of inflationary models. Here the idea is that quantum fluctuations of an Abelian gauge field (for instance the hypercharge field) are inside the Hubble radius during inflation. Then, these fluctuations exit the horizon and their correlation scale grows faster than the Hubble radius itself. The amount of amplification achieved in this processes depends crucially on the amount of breaking of conformal invariance of the evolution equation of the Abelian gauge fields. A lot of ideas concerning the possible breaking of conformal invariance (see, for instance, 63. and [80]) have been proposed so far and it would be impossible to summarize them all in this topical review.

Instead going through the various ideas it may be more useful to give further details about a specific way of breaking conformal invariance like the one suggested in the context of string cosmological models [86]. The string effective action contains a direct coupling between the (scalar) dilaton field and the electromagnetic field that appears even at tree level in the loop expansion and to lowest order in the string tension expansion. To lowest order the action will then be

$$
S=-\frac{1}{4} \int d^{4} x \sqrt{-g} e^{-\varphi} Y_{\alpha \beta} Y^{\alpha \beta},
$$

where $Y_{\alpha \beta}$ is the field strength. If the gauge coupling evolves during the inflationary dynamics, then the magnetic fields will be amplified over different length-scales. In particular, in the model presented in [86], the gauge coupling, i.e. $e^{\varphi / 2}$ is a growing function of the cosmic time coordinate. When the Universe becomes dominated by radiation (after inflation) the gauge coupling freezes to a value that is of order of $10^{-2}$. More specifically, there are two phases that characterize the model. In the first phase the gauge coupling increases sharply 
(the so-called dilaton driven phase). In the second phase the gauge coupling is just slowly increasing (the so-called stringy phase).

In the context of string cosmological models there are two possible ranges in the values of $m$, i.e. the spectral index of the produced magnetic fields. If the relevant modes (i.e. comoving scales larger than the Mpc scale) exit the Hubble radius for the first time during the so-called dilaton driven phase, the resulting spectrum leads to an $m$ that could be written as 86

$$
m=-\frac{\sqrt{3}+3 \sqrt{1-\Sigma}}{\sqrt{3}+\sqrt{1-\Sigma}}
$$

where $\Sigma=\sum_{i} \beta_{i}^{2}$ represents the possible effect of internal dimensions whose radii $b_{i}$ shrink, during the pre-big bang phase, like $(-t)^{\beta_{i}}$ for $t \rightarrow 0^{-}$. In the case $\Sigma=0$ we will have $m \sim-\sqrt{3}$.

The second possibility is that the relevant large-scale modes left the Hubble radius during the so-called string phase. During this phase the ratio between the (cosmic) time derivative of the dilaton field and the Hubble rate is roughly constant. Defining as $\beta$ the mentioned ratio $\dot{\varphi} / 2 H$, we will have that $m \simeq 1-2 \beta$. If the value of $\beta$ gets frozen at the value corresponding to the last e-folds of the pre-big bang phase we will have, for instance, allowed values between $\beta \sim 1.8$ and $\beta \sim 2$ corresponding to $m \simeq-3$ (i.e. $-2.9,-2.8$ ). This parameter can be computed once a specific model of the high-curvature (stringy) regime is given but it cannot be obtained, at present, from more general arguments. Notice that, in this case, $B_{L}$ may even be of the order of $10^{-10}$ corresponding to a ratio between the magnetic and radiation energy density of the order of $10^{-8}$ [86].

It is also possible to generate large scale magnetic fields within the Abelian Higgs model where the Higgs current densities allow to obtain a (minute) amplification of the hypermagnetic field. The amplitude of these fields is irrelevant for applications (i.e. the produced field intensity is thirty orders of magnitude smaller than the most optimistic dynamo requirements). The typical spectral slope is $m=1$ that corresponds to the magnetic spectrum of the vacuum once the canonical quantum mechanical normalization is imposed on the vector potentials. Slightly different spectra can be obtained in the case of the models proposed in [87, 88, 89] where various charged scalars are considered together (see also [90]).

It is appropriate to comment here on the possibility that large-scale magnetization is a purely astrophysical phenomenon. As in the case of the primordial hypothesis, it is rather difficult to summarize, in a reasonably self-contained manner all the hypothesis that have been put forward in the last fifty years. An (incomplete) list of proposals can be found, for instance in 63, 91, 92. The modest aim of the remaining part of this section will therefore be to introduce the general framework. 
Many (if not all) the astrophysical mechanisms proposed so far are related to what is called, in the jargon, a battery. The idea is, in short the following. MHD, as previously stressed in this section, can be "derived" from a two-fluid theory by appropriately defined one-fluid variables that obey a set of effective Maxwell equations supplemented by the Ohm law (i.e. Eq. (4.5)) and coupled to the Navier-Stokes equation. Now, we already remarked right before Eq. (4.5) that the proposed form of the Ohmic electric field would contain, in principle, also a thermoelectric term that is a remnant of the subtraction of the momentum conservation equations that hold separately (for electrons and ions) in the twofluid description from which MHD is derived. The explicit form of the generalized Ohmic electric field in the presence of thermoelectric corrections can be written as ${ }^{13}$

$$
\vec{E}=-\vec{v} \times \vec{B}+\frac{\vec{\nabla} \times \vec{B}}{4 \pi \sigma}-\frac{\vec{\nabla} P_{\mathrm{e}}}{e n_{\mathrm{e}}} .
$$

By comparing Eq. (4.5) with Eq. (4.35), it is clear that the additional term at the right hand side, receives contribution from a temperature gradient. In fact, restoring for a moment the Boltzmann constant $k_{B}$ we have that since $P_{\mathrm{e}}=k_{B} n_{\mathrm{e}} T_{\mathrm{e}}$, the additional term depends upon the gradients of the temperature, hence the name thermoelectric. It is interesting to see under which conditions the curl of the electric field receives contribution from the thermoelectric effect. Taking the curl of both sides of Eq. (4.35) we obtain

$$
\vec{\nabla} \times \vec{E}=\frac{1}{4 \pi \sigma} \nabla^{2} \vec{B}+\vec{\nabla}(\vec{v} \times \vec{B})-\frac{\vec{\nabla} n_{\mathrm{e}} \times \vec{\nabla} P_{\mathrm{e}}}{e n_{\mathrm{e}}^{2}}=-\frac{\partial B}{\partial t},
$$

where the second equality is a consequence of Maxwell's equations. From Eq. (4.36) it is clear that the evolution of the magnetic field inherits a source term iff (i.e. if and only if) the gradients in the pressure and electron density are not parallel. If $\vec{\nabla} P_{\mathrm{e}} \| \vec{\nabla} n_{\mathrm{e}}$ a fully valid solution of Eq. (4.36) is $\vec{B}=0$. In the opposite case a seed magnetic field is naturally provided by the thermoelectric term. The usual (and rather general) observation that one can make in connection with the geometrical properties of the thermoelectric term is that cosmic ionization fronts may play an important rôle. For instance, when quasars emit ultraviolet photons, cosmic ionization fronts are produced. Then the intergalactic medium may be ionized. It should also be recalled, however, that the temperature gradients are usually normal to the ionization front. In spite of this, it is also plausible to think that density gradients can arise in arbitrary directions due to the stochastic nature of density fluctuations.

\footnotetext{
${ }^{13}$ For simplicity, we shall neglect the Hall contribution arising in the generalized Ohm law. The Hall contribution would produce, in Eq. 4.35 a term $\vec{J} \times \vec{B} / n_{\mathrm{e}} e$ that is of higher order in the magnetic field and that is proportional to the Lorentz force. The Hall term will play no rôle in the subsequent considerations. However, it should be borne in mind that the Hall contribution may be rather interesting in connection with the presence of strong magnetic fields like the ones of neutron stars (i.e. $10^{13} \mathrm{G}$ ). This occurrence is even more interesting since in the outer regions of neutron stars strong density gradients are expected.
} 
In one way or in another, astrophysical mechanisms for the generation of magnetic fields use an incarnation of the thermoelectric effect. In the sixties and seventies, for instance, it was rather popular to think that the correct "geometrical" properties of the thermoelectric term may be provided by a large-scale vorticity. As it will also be discussed later, this assumption seems to be, at least naively, in contradiction with the formulation of inflationary models whose prediction would actually be that the large-scale vector modes are completely washed-out by the expansion of the Universe. Indeed, all along the eighties and nineties the idea of primordial vorticity received just a minor attention.

The attention then focused on the possibility that objects of rather small size may provide intense seeds. After all we do know that these objects may exist. For instance the Crab nebula has a typical size of a roughly $1 \mathrm{pc}$ and a magnetic field that is a fraction of the $\mathrm{m}$ G. These seeds will then combine and diffuse leading, ultimately, to a weaker seed but with large correlation scale. This aspect, may be, physically, a bit controversial since we do observe magnetic fields in galaxies and clusters that are ordered over very large length scales. It would then seem necessary that the seed fields produced in a small object (or in several small objects) undergo some type of dynamical self-organization whose final effect is a seed coherent over length-scales 4 or 5 orders of magnitude larger than the correlation scale of the original battery.

An interesting idea could be that qualitatively different batteries lead to some type of conspiracy that may produce a strong large scale seed. In 92 it has been suggested that Population III stars may become magnetized thanks to a battery operating at stellar scale. Then if these stars would explode as supernovae (or if they would eject a magnetized stellar wind) the pre-galactic environment may be magnetized and the remnants of the process incorporated in the galactic disc. In a complementary perspective, a similar chain of events may take place over a different physical scale. A battery could arise, in fact in active galactic nuclei at high red-shift. Then the magnetic field could be ejected leading to intense fields in the lobes of "young" radio-galaxies. These fields will be somehow inherited by the "older" disc galaxies and the final seed field may be, according to [92 as large as $10^{-9} \mathrm{G}$ at the pre-galactic stage.

Up to now only a necessarily reduced account of the primordial and of the astrophysical hypotheses has been presented. In spite of the mechanism leading to a seed field, the crucial problem will be how to go from the seed to the actual galactic (or even cluster) magnetic field. The galactic rotation period is of the order of $3 \times 10^{8}$ yrs. This scale should be compared with the typical age of the galaxy. All along this rather large dynamical time-scale the effort has been directed, from the fifties, to the justification that a substantial portion of the kinetic energy of the system (provided by the differential rotation) may be converted 
into magnetic energy amplifying, in this way, the seed field up to the observed value of the magnetic field, for instance in galaxies and in clusters. In recent years a lot of progress has been made both in the context of the small and large-scale dynamos. This progress was also driven by the higher resolution of the numerical simulations and by the improvement in the understanding of the largest magnetized system that is rather close to us, i.e. the sun. More complete accounts of this progress can be found in the second paper of Ref. 93] and, more comprehensively, in Ref. 94. The main ingredients of the large-scale dynamo can be also found in 63. Apart from the aspects involving solar physics and numerical analysis, better physical understanding of the rôle of the magnetic helicity in the dynamo action has been reached. This point is crucially connected with the two conservation laws arising in MHD, i.e. the magnetic flux and magnetic helicity conservations whose relevance has been already emphasized, respectively, in Eqs. (4.6) and (4.7). Even if the rich interplay between small and large scale dynamos is rather important, let us focus on the problem of large-scale dynamo action that is, at least superficially, more central for the considerations developed in the present paper. In short, the picture of large-scale dynamos can be summarized as follows. In a turbulent environment the MHD equations can be appropriately averaged with the result that the mean magnetic seed is amplified exponentially fast. This approach is described in detail in 94. (see also 95]). The most important point to bear in mind is that the turbulent velocity field (whose correlation scale is typically shorter than the correlation scale of the magnetic seed) must lack mirror symmetry. In other words the velocity field must be parity-odd. This occurrence necessarily implies that the (averaged) magnetic diffusivity equation inherits a term that is proportional to the curl of the magnetic field though a coefficient, the celebrated $\alpha$ term, that would vanish if the velocity field would be parity-even. It can be demonstrated that the $\alpha$ term is indeed proportional, in turn, to the average kinetic helicity, i.e. $\langle\vec{v} \cdot \vec{\nabla} \times \vec{v}\rangle$. The $\alpha$ term decreases more slowly, at large length-scales, than the diffusivity terms. Therefore, for sufficiently large scales the magnetic seed can be efficiently (exponentially?) amplified. For long time this oversimplified picture was thought to be essentially consistent even if computationally challenging.

Already at a qualitative level it is clear that there is a clash between the absence of mirror-symmetry of the plasma, the quasi-exponential amplification of the seed and the conservation of magnetic flux and helicity in the high (or more precisely infinite) conductivity limit. The easiest clash to understand, intuitively, is the flux conservation versus the exponential amplification: both flux freezing and exponential amplification have to take place in the same superconductive (i.e. $\sigma^{-1} \rightarrow 0$ ) limit. The clash between helicity conservation and dynamo action can be also understood in general terms: the dynamo action implies a topology change of the configuration since the magnetic flux lines cross each other constantly 93 . 
One of the recent progress in this framework is a more consistent formulation of the large-scale dynamo problem 93, 94]: large scale dynamos produces small scale helical fields that quench (i.e. prematurely saturate) the $\alpha$ effect. In other words, the conservation of the magnetic helicity can be seen, according to the recent view, as a fundamental constraint on the dynamo action. In connection with the last point, it should be mentioned that, in the past, a rather different argument was suggested [96]: it was argued that the dynamo action not only leads to the amplification of the large-scale field but also of the random field component. The random field would then suppress strongly the dynamo action. According to the considerations based on the conservation of the magnetic helicity this argument seems to be incorrect since the increase of the random component would also entail and increase of the rate of the topology change, i.e. a magnetic helicity non-conservation.

In summary we can therefore say that:

- both the primordial and the astrophysical hypothesis for the origin of the seeds demand an efficient (large-scale) dynamo action;

- due to the constraints arising from the conservation of magnetic helicity and magnetic flux the values of the required seed fields may turn out to be larger than previously thought at least in the case when the amplification is only driven by a large-scale dynamo action ${ }^{14}$;

- magnetic flux conservation during gravitational collapse of the protogalaxy may increase, by compressional amplification, the initial seed of even 4 orders of magnitude;

- compressional amplification, as well as large-scale dynamo, are much less effective in clusters: therefore, the magnetic field of clusters is probably connected to the specific way the dynamo saturates, and, in this sense, harder to predict from a specific value of the initial seed.

\section{$5 \quad$ Magnetized metric perturbations}

Consider a conformally flat background geometry

$$
\bar{g}_{\mu \nu}=a^{2}(\tau) \eta_{\mu \nu}
$$

where $\eta_{\mu \nu}=\operatorname{diag}(1,-1,-1,-1)$ is the flat Minkowski metric. Then the fluctuations of the metric may be written as

$$
\delta g_{\mu \nu}=\delta_{\mathrm{t}} g_{\mu \nu}+\delta_{\mathrm{v}} g_{\mu \nu}+\delta_{\mathrm{s}} g_{\mu \nu}
$$

\footnotetext{
${ }^{14}$ The situation may change if the magnetic fields originate from the combined action of small and large scale dynamos like in the case of the two-step process described in 92 .
} 
where $\delta_{\mathrm{t}}, \delta_{\mathrm{v}}$ and $\delta_{\mathrm{s}}$ denote the tensor, vector and scalar fluctuation of the geometry.

The evolution of the various modes of the geometry are sourced by the corresponding modes of the energy-momentum tensor of the fluid sources and of the magnetic field. In particular, denoting with $\mathcal{T}_{\mu \nu}$ the energy-momentum tensor of the magnetic field we will have, following the notation of Eq. (5.2), that

$$
\delta \mathcal{T}_{\mu \nu}=\delta_{\mathrm{t}} \mathcal{T}_{\mu \nu}+\delta_{\mathrm{v}} \mathcal{T}_{\mu \nu}+\delta_{\mathrm{s}} \mathcal{T}_{\mu \nu}
$$

The fluctuations $\delta \mathcal{T}_{\mu \nu}$ are quadratic in the intensity of the fully inhomogeneous magnetic fields. This means, that a non-Gaussian signal should be, in general, expected. The rationale for this statement is that while the fluctuations of fully inhomogeneous magnetic fields may be Gaussian (see, for instance, Eq. (4.9)), the fluctuations of the energy-momentum tensor will be distributed as the square of a Gaussian variable and will therefore be non-Gaussian. Recently, more accurate calculations of the possible non-Gaussian effects arising in the presence of fully inhomogeneous magnetic fields have been presented [97.

In the following the evolution equations of tensor, vector and scalar modes will be treated within a (first-order) "Bardeen" approach [98] (see also [40] for an introduction to the key features of the problem in the absence of magnetic fields). The evolution of fully inhomogeneous magnetic fields can be usefully treated also within a covariant formalism. For this type of analysis see the interesting studies reported in Refs. 99, 100, 101.

\subsection{Magnetized tensor modes}

The tensor fluctuations of the metric defined as

$$
\delta_{\mathrm{t}} g_{i j}=-a^{2} h_{i j}, \quad \partial_{i} h_{j}^{i}=0, \quad h_{i}^{i}=0,
$$

are automatically invariant under infinitesimal coordinate transformations. Equation (5.4) implies that $h_{i j}$ carries two independent degrees of freedom. From the fluctuation of the Ricci tensor it is easy to obtain that the evolution equations are

$$
h_{i}^{j^{\prime \prime}}+2 \mathcal{H} h_{i}^{j^{\prime}}-\nabla^{2} h_{i}^{j}=-16 \pi G a^{2} \delta_{\mathrm{t}} \mathcal{T}_{i}^{j}
$$

where, as mentioned before, $\delta_{\mathrm{t}} \mathcal{T}_{i}^{j}$ is the transverse and traceless component of the energymomentum tensor of the electromagnetic fields. Given the specific magnetic field configuration, Eq. (5.5) allows to determine the amplitude of the gravitational waves. The determination of $h_{i j}$ allows the determination of the tensor Sachs-Wolfe contribution, i.e.

$$
\left(\frac{\Delta T}{T}\right)_{\mathrm{t}}=-\frac{1}{2} \int_{\tau_{i}}^{\tau_{f}} h_{i j}^{\prime} n^{i} n^{j} d \tau
$$


In the tensor case the fluctuations of the geometry do not mix with the fluctuations of the fluid sources. Furthermore, in the tensor case, as it is well known, only the Sachs-Wolfe integral contributes. Tensor modes have been discussed in a number of papers. In [30] and 102 the possible stochastic backgrounds of gravitational radiation stemming from inhomogeneous magnetic fields have been computed with particular attention to configurations carrying non-vanishing magnetic gyrotropy. Recently gravitational waves produced through a phase of helical turbulence have been discussed in [103]. For a discussion of the interplay of gravitational waves and inhomogeneous magnetic fields see also [104.

\subsection{Magnetized vector modes}

The evolution equations arising in the case of vector modes are a bit more complicated than in the case of tensor modes. The vector modes of the geometry are parametrized in the following way

$$
\delta_{\mathrm{v}} g_{0 i}=-a^{2} Q_{i}, \quad \delta_{\mathrm{v}} g_{i j}=a^{2}\left(\partial_{i} W_{j}+\partial_{j} W_{i}\right),
$$

where $\partial_{i} Q^{i}=0$ and $\partial_{i} W^{i}=0$.

The perturbed Einstein and covariant conservation equations read

$$
\begin{gathered}
\delta_{\mathrm{v}} R_{\mu}^{\nu}=8 \pi G\left(\delta_{\mathrm{v}} T_{\mu}^{\nu}+\delta_{\mathrm{v}} \mathcal{T}_{\mu}^{\nu}\right), \\
\nabla_{\mu} \delta_{\mathrm{v}} T^{\mu \nu}+\nabla_{\mu} \delta_{\mathrm{v}} \mathcal{T}^{\mu \nu}=0,
\end{gathered}
$$

where

$$
\begin{aligned}
\delta_{\mathrm{v}} T_{\mu}^{\nu} & =(p+\rho)\left(u_{\mu} \delta_{\mathrm{v}} u^{\nu}+\delta_{\mathrm{v}} u_{\mu} u^{\nu}\right), \\
\delta_{\mathrm{v}} \mathcal{T}_{\mu}^{\nu} & =\frac{1}{4 \pi}\left(-F_{\mu \alpha} F^{\nu \alpha}+\frac{1}{4} \delta_{\mu}^{\nu} F_{\alpha \beta} F^{\alpha \beta}\right) .
\end{aligned}
$$

Equations (5.8) and (5.11) have to be supplemented by Maxwell's equations together with the generalized Ohm's law

$$
\begin{aligned}
& \nabla_{\mu} F^{\mu \nu}=4 \pi j^{\mu} \\
& j^{\mu}=\sigma_{\mathrm{c}} F^{\mu \nu} u_{\nu}
\end{aligned}
$$

Recall first that

$$
\begin{aligned}
\delta_{\mathrm{v}} R_{0}^{i} & =\frac{2}{a^{2}} Q^{i}\left(\mathcal{H}^{\prime}-\mathcal{H}^{2}\right)+\frac{1}{2 a^{2}} \nabla^{2} V^{i}, \\
\delta_{\mathrm{v}} R_{i}^{j} & =\frac{1}{2 a^{2}}\left[\left(\partial_{i} V^{j}+\partial^{j} V_{i}\right)^{\prime}+2 \mathcal{H}\left(\partial_{i} V^{j}+\partial^{j} V_{i}\right)\right] .
\end{aligned}
$$

where

$$
\vec{V}=\vec{Q}+\vec{W}^{\prime}
$$


The vector $\vec{V}$ is gauge-invariant. In fact, for infinitesimal gauge transformations preserving the vector nature of the fluctuation we have, indeed,

$$
\begin{aligned}
& Q_{i} \rightarrow \tilde{Q}_{i}=Q_{i}-\zeta_{i}^{\prime}, \\
& W_{i} \rightarrow \tilde{W}_{i}=W_{i}+\zeta_{i}
\end{aligned}
$$

From Eq. (5.10), recalling that

$$
u_{0} \delta_{\mathrm{v}} u^{i}=-\left(\mathcal{V}^{i}+Q^{i}\right)
$$

we simply have

$$
\delta_{\mathrm{v}} T_{0}^{i}=-(p+\rho)\left(\mathcal{V}^{i}+Q^{i}\right) .
$$

Equation (5.11) become, in explicit terms,

$$
\begin{aligned}
& \delta_{\mathrm{v}} \mathcal{T}_{0}^{i}=\frac{1}{4 \pi}(\overrightarrow{\mathcal{E}} \times \overrightarrow{\mathcal{B}})^{i} \equiv \frac{1}{4 \pi a^{4}}(\vec{E} \times \vec{B})^{i}, \\
& \delta_{\mathrm{v}} \mathcal{T}_{i}^{j}=\frac{1}{4 \pi}\left[\mathcal{E}_{i} \mathcal{E}^{j}+\mathcal{B}_{i} \mathcal{B}^{j}-\frac{1}{2} \delta_{i}^{j}\left(\overrightarrow{\mathcal{B}}^{2}+\overrightarrow{\mathcal{E}}^{2}\right)\right] \\
& \equiv \frac{1}{4 \pi a^{4}}\left[E_{i} E^{j}+B_{i} B^{j}-\frac{1}{2} \delta_{i}^{j}\left(\vec{B}^{2}+\vec{E}^{2}\right)\right],
\end{aligned}
$$

where the following conventions have been adopted

$$
\begin{aligned}
& F_{0 i}=a^{2} \mathcal{E}_{i}, \quad F^{0 i}=-\frac{1}{a^{2}} \mathcal{E}^{i}, \\
& F_{i j}=-a^{2} \epsilon_{i j k} \mathcal{B}^{k}, \quad F^{i j}=-\frac{1}{a^{2}} \epsilon^{i j k} \mathcal{B}_{k}, \\
& \vec{E}=a^{2} \overrightarrow{\mathcal{E}}, \quad \vec{B}=a^{2} \overrightarrow{\mathcal{B}} .
\end{aligned}
$$

Therefore, the perturbed set of equations can be written, in the gauge $W_{i}=0$,

$$
\begin{aligned}
& \nabla^{2} \vec{Q}=-16 \pi G a^{2}(p+\rho) \overrightarrow{\mathcal{V}}+\frac{16 \pi G}{\sigma a^{2}} \vec{F}_{B}(\vec{x}), \\
& \nabla^{2}\left(\vec{Q}^{\prime}+2 \mathcal{H} \vec{Q}\right)=\frac{16 \pi G}{a^{2}} \vec{F}_{B}(\vec{x}), \\
& \overrightarrow{\mathcal{V}}^{\prime}+\left[4 \mathcal{H}+\frac{p^{\prime}+\rho^{\prime}}{p+\rho}\right] \overrightarrow{\mathcal{V}}+\frac{\vec{F}_{B}(\vec{x})}{a^{4}(p+\rho)}=0,
\end{aligned}
$$

where

$$
\vec{F}_{B}=\frac{1}{4 \pi}(\vec{\nabla} \times \vec{B}) \times \vec{B}
$$

Notice that the divergence-full part of the Lorentz force (i.e. $\vec{\nabla} \cdot \vec{F}_{B}$ ) does not contribute to the evolution equations of the vectors but it does contribute to the evolution equations 
of the scalar modes. The second term at the right hand side of Eq. (5.23) contains, in the denominator, $\sigma=a \sigma_{\mathrm{c}}$, i.e. the rescaled conductivity. This term, coming from the MHD form of the Poynting vector, is negligible in the infinite conductivity limit and it is usually dropped.

Formally, the solution of the above system of equations can be written as

$$
\begin{aligned}
& \vec{V}(\vec{x}, \eta)=-\frac{1}{a^{4}(\eta)(p+\rho)} \int \vec{F}_{B}(\vec{x}, \eta) d \eta-\frac{\vec{C}(\vec{x})}{16 \pi G a^{4}(\eta)(p+\rho)}, \\
& \nabla^{2} \vec{Q}=\frac{16 \pi G}{a^{2}(\eta)} \int \vec{F}_{B}(\vec{x}, \eta) d \eta+\frac{\vec{C}(\vec{x})}{a^{2}(\eta)} .
\end{aligned}
$$

Finally, the vector contribution to the Sachs-Wolfe effect can be written, in the gauge $W_{i}=0$ as

$$
\left(\frac{\Delta T}{T}\right)_{\mathrm{v}}=[-\overrightarrow{\mathcal{V}} \cdot \vec{n}]_{\tau_{i}}^{\tau_{f}}+\frac{1}{2} \int_{\tau_{i}}^{\tau_{f}}\left(\partial_{i} Q_{j}+\partial_{j} Q_{i}\right) n^{i} n^{j} d \tau
$$

In the absence of large-scale magnetic field, the only solution of the above system is the one denoted by the integration constant $C$. In this case, if the Universe expands, the vector modes can only decay (see, however, 105, 106, 107]).

In a series of papers Barrow and Subramanian [108, 109] analyzed the effects of tangled magnetic fields both on the temperature and polarization anisotropies. More recently the vector modes have been analyzed numerically in Ref. [110] (see also [111]). The obtained results are in qualitative agreement with the semi-analytical estimates presented in 108 , 109. In [110] the effect of the neutrinos has been accurately parametrized in the evolution equations. In the equations for the vector modes written above, the (divergenceless) velocity field $\overrightarrow{\mathcal{V}}$ has been taken generic. However, when the plasma content before decoupling is specified, $\overrightarrow{\mathcal{V}}$, i.e. the total velocity field will receive contribution from all the species present in the plasma. A similar situation will be encountered in the case of scalar modes and will be briefly discussed there.

\subsection{Magnetized scalar modes}

The evolution of scalar fluctuations is, technically, more complicated than the vector and tensor problems. In the case of scalar fluctuations, the magnetic fields enter the evolution equations through the magnetic energy density and through the divergence of the Lorentz force. Furthermore, in the scalar case, the possible gauge choices increase with respect to the case of the vector modes.

In the absence of magnetic fields, we do know that the most general initial conditions for CMB anisotropies consist of one adiabatic mode and four non-adiabatic (or isocurvature) 
modes (see, for instance, [40]). In the case of adiabatic modes the fluctuations in the specific entropy vanish for typical length-scales larger than the Hubble radius. This occurrence implies a specific relation among the density contrasts of the various species present in the plasma. Consider, indeed, the simple case of a system formed by cold dark matter (CDM) and radiation. In this case, the specific entropy $\varsigma$ and its relative fluctuations $\mathcal{S}$ can be written as

$$
\varsigma=\frac{T^{3}}{n_{\mathrm{c}}}, \quad \mathcal{S}=\frac{\delta \varsigma}{\varsigma}=\frac{3}{4} \delta_{\gamma}-\delta_{\mathrm{c}}
$$

where $n_{\mathrm{c}}$ is the number density of CDM particles; $\delta_{\gamma}$ and $\delta_{\mathrm{c}}$ are the density contrasts of the fluctuations in radiation and in $\mathrm{CDM}$. If $\mathcal{S} \simeq 0$ for scales larger than the Hubble radius, the fluctuations are said to be adiabatic.

This naive definition can be generalized in various ways. One can, for instance, give a more general gauge-invaraint definition by introducing two generic fluids, the a-fluid and the b-fluid. In this case it is rather natural to define the two gauge-invariant variables

$$
\begin{gathered}
\zeta_{\mathrm{a}}=-\psi-\mathcal{H} \frac{\delta \rho_{\mathrm{a}}}{\rho_{\mathrm{a}}^{\prime}}, \\
\zeta_{\mathrm{b}}=-\psi-\mathcal{H} \frac{\delta \rho_{\mathrm{b}}}{\rho_{\mathrm{b}}^{\prime}},
\end{gathered}
$$

that can be interpreted, respectively, as the curvature fluctuations on uniform a-density and b-density hypersurfaces (see, for instance, [112 and references therein). The (total) curvature fluctuations on uniform density hypersurfaces are then

$$
\zeta=\frac{\rho_{\mathrm{a}}^{\prime}}{\rho^{\prime}} \zeta_{\mathrm{a}}+\frac{\rho_{\mathrm{b}}^{\prime}}{\rho^{\prime}} \zeta_{\mathrm{b}} \equiv-\psi-\mathcal{H} \frac{\delta \rho}{\rho^{\prime}}
$$

where $\rho=\rho_{\mathrm{a}}+\rho_{\mathrm{b}}$. From Eqs. (5.30) and (5.31) it is possible to define the generalized entropy fluctuations as

$$
\mathcal{S}=-3\left(\zeta_{\mathrm{b}}-\zeta_{\mathrm{a}}\right)=\frac{\delta_{\mathrm{b}}}{1+w_{\mathrm{b}}}-\frac{\delta_{\mathrm{a}}}{1+w_{\mathrm{a}}},
$$

where the second equality follows from the first equality by using the covariant conservation equation for the each of the two fluids; $w_{\mathrm{a}}$ and $w_{\mathrm{b}}$ are, respectively, the barotropic indices for the a-fluid and the b-fluid. In the case $w_{\mathrm{b}}=w_{\gamma}=1 / 3$ and $w_{\mathrm{a}}=0$ Eq. (5.33) reproduces Eq. (5.29).

If we now consider the plasma after equality but before decoupling, the total entropy fluctuations will vanish iff

$$
\delta_{\gamma} \simeq \delta_{\nu} \simeq \frac{4}{3} \delta_{\mathrm{c}} \simeq \frac{4}{3} \delta_{\mathrm{b}}
$$

where the density contrast in the neutrinos and in the baryons, i.e. $\delta_{\nu}$ and $\delta_{\mathrm{b}}$, have been introduced. 
If magnetic fields are included in the game, then the adiabatic mode, together with the four isocurvature modes, can be generalized. The logic will then be the following:

- the evolution equations of the whole system must be written including the contribution of the large-scale magnetic fields;

- the system includes, on top of the magnetic field and of the scalar fluctuations of the geometry also the neutrinos, the photons, the baryons and the CDM particles;

- the equations must be solved when the relevant modes are larger than the Hubble radius;

- the obtained solutions will serve as initial conditions for the lowest multipoles of the Boltzmann hierarchy.

It is difficult, in the scalar case, to go trough all the previous steps without changing gauge. Indeed it is well known, also in the absence of large-scale magnetic fields, that some isocurvature modes (for which the presence of anisotropic stresses is crucial) are regular in the synchronous gauge but are divergent (at early times) in the longitudinal gauge. This is just an example and, in the following, the magnetized adiabatic mode will be mainly discussed, quoting, when appropriate, some results of more technical investigations [113].

\subsubsection{Magnetized adiabatic mode}

Consider, for simplicity, the system of scalar fluctuations in the longitudinal gauge where the metric fluctuations can be written in terms of the two functions $\phi$ and $\psi$, i.e.

$$
\delta_{\mathrm{s}} g_{00}=2 a^{2} \phi, \quad \delta_{\mathrm{s}} g_{i j}=2 a^{2} \psi \delta_{i j}
$$

In the following, the relevant equations of the system will be discussed starting with the evolution equations of the fluctuations. As mentioned before, the plasma content is assumed to be formed by neutrinos, photons, baryons, CDM particles and fully inhomogeneous maggnetic fields.

In the gauge of Eq. (5.35), the Hamiltonian constraint reads

$$
-3 \mathcal{H}\left(\mathcal{H} \phi+\psi^{\prime}\right)-k^{2} \psi=\frac{3}{2} \mathcal{H}^{2}\left[\left(R_{\nu} \delta_{\nu}+\left(1-R_{\nu}\right) \delta_{\gamma}\right)+\Omega_{\mathrm{B}}(k)+\Omega_{\mathrm{b}} \delta_{\mathrm{b}}+\Omega_{\mathrm{c}} \delta_{\mathrm{c}}\right],
$$

where, for $N_{\nu}$ species of massless neutrinos,

$$
R=\frac{7}{8} N_{\nu}\left(\frac{4}{11}\right)^{4 / 3}, \quad R_{\nu}=\frac{R}{1+R}, \quad R_{\gamma}=1-R_{\nu}
$$


so that $R_{\nu}$ and $R_{\gamma}$ represent the fractional contributions of photons and neutrinos to the total density at early times deep within the radiation-dominated epoch.

In Eq. (5.36) the contribution of the magnetic energy density has been parametrized, in Fourier space, as

$$
\Omega_{\mathrm{B}}(k, \eta)=\frac{\rho_{\mathrm{B}}}{\rho}=\frac{1}{8 \pi \rho a^{4}} \int d^{3} p B_{i}(|\vec{p}-\vec{k}|) B^{i}(p) .
$$

The appearance of convolutions in the expressions of the magnetic energy density is a direct consequence of the absence of a uniform component of the magnetic field whose background contribution, as repeatedly stressed, is vanishing. Notice also that $\rho$ appearing in Eq. (5.38) is the energy density of the dominant component of the fluid, so, for instance, in the radiation-dominated epoch $\rho \equiv \rho_{\gamma}, a^{4} \rho_{\gamma} \sim$ constant and $\Omega_{B} \simeq$ constant.

While the Hamiltonian constraint relates the metric fluctuations (and their first time derivatives) to the density contrasts of the various species, the momentum constraints relates the metric fluctuations (and their first derivatives) to the divergence-full part of the peculiar velocity field and to the MHD expression of the Poynting vector ${ }^{15}$ :

$$
k^{2}\left(\mathcal{H} \phi+\psi^{\prime}\right)=\frac{3}{2} \mathcal{H}^{2}\left[\frac{4}{3}\left(R_{\nu} \theta_{\nu}+\left(1-R_{\nu}\right) \theta_{\gamma}\right)+\frac{\mathcal{F}_{\mathrm{B}}(k)}{4 \pi \sigma a^{4} \rho}+\theta_{\mathrm{b}} \Omega_{\mathrm{b}}+\theta_{\mathrm{c}} \Omega_{\mathrm{c}}\right],
$$

where $\mathcal{F}_{\mathrm{B}}(k, \eta)$ is the Fourier transform of the generalized Lorentz force, i.e.

$$
\vec{\nabla} \cdot[(\vec{\nabla} \times \vec{B}) \times \vec{B}]=\int d^{3} k \mathcal{F}_{\mathrm{B}}(k) e^{i \vec{k} \cdot \vec{x}}
$$

and $\mathcal{F}_{\mathrm{B}}(k)$ is given by the following convolution

$$
\int d^{3} p\left[(\vec{k} \cdot \vec{p}) B_{j}(p) B^{j}(|\vec{k}-\vec{p}|)-\left(k_{i}-p_{i}\right) B_{j}(|\vec{k}-\vec{p}|) B_{j}(|\vec{k}-\vec{p}|) B^{j}(p)\right] .
$$

Notice that a consistent treatment of fully inhomogeneous magnetic fields implies that

$$
\Omega_{\mathrm{B}} \ll 1, \quad \frac{\mathcal{F}_{\mathrm{B}}}{4 \pi k^{2} \rho a^{4}} \ll 1,
$$

in order not to over-close the Universe. In the ideal MHD limit, i.e. $\sigma \rightarrow \infty$, the contribution of $\mathcal{F}_{\mathrm{B}}(k)$ disappears from Eq. (5.39).

Another important condition on the difference of the conformally Newtonian potentials comes from the $(i \neq j)$ components of the perturbed Einstein equations:

$$
k^{2}(\phi-\psi)=\frac{9}{2} \mathcal{H}^{2}\left[-\frac{4}{3} \sigma_{\nu} R_{\nu}-\sigma_{\mathrm{B}}(k)\right]
$$

\footnotetext{
${ }^{15}$ In the following $\theta_{\gamma}=\partial_{i} v_{\gamma}^{i}, \theta_{\mathrm{c}}=\partial_{i} v_{\mathrm{c}}^{i}$ and so on for the two remaining species, i.e. $\theta_{\mathrm{b}}$ and $\theta_{\nu}$.
} 
having defined

$$
\sigma_{\mathrm{B}}=\frac{\Omega_{\mathrm{B}}(k)}{3}-\frac{\mathcal{F}_{\mathrm{B}}(k)}{4 \pi a^{4} \rho k^{2}} .
$$

The quantity $\sigma_{\mathrm{B}}$ can be interpreted as the anisotropic stress arising thanks to the inhomogeneous magnetic field and, in the force-free case, $\sigma_{\mathrm{B}}=\Omega_{\mathrm{B}} / 3$. Equation (5.43) relates the difference of the two metric fluctuations to a combination of the neutrino and magnetic anisotropic stresses.

Let us finally come to the equation stemming from the perturbed $(i=j)$ components of the Einstein equations:

$$
\psi^{\prime \prime}+\left(2 \psi^{\prime}+\phi^{\prime}\right) \mathcal{H}+\left(2 \mathcal{H}^{\prime}+\mathcal{H}^{2}\right) \phi-\frac{k^{2}}{3}(\phi-\psi)=\frac{1}{2} \mathcal{H}^{2}\left[\left(R_{\nu} \delta_{\nu}+\left(1-R_{\nu}\right)\right) \delta_{\gamma}+\Omega_{\mathrm{B}}(k)\right]
$$

where the contribution of the other species vanishes since both baryons and CDM particles have a vanishing barotropic index, i.e. $w_{\mathrm{b}}=w_{\mathrm{c}}=0$.

Equations (5.36), (5.39), (5.43) and (5.45) should be supplemented by the evolution equations of the various plasma quantities. For doing so, there are two complementary approaches. In the first approach, one may notice that the perturbed Einstein equations only involve the density contrasts and the peculiar velocities of the various species, i.e. the monopoles and the dipoles of the (perturbed) phase spece distributions. Therefore, the simplest approach is to describe the evolution of the different species of the plasma through an appropriate set of fluid equations (following from the covariant conservation equations for each species). The exception in this statement is represented by the neutrinos whose evolution includes also the quadrupole of the neutrino phase space distribution, i.e. $\sigma_{\nu}$. To treat neutrinos one should include, therefore, also higher multipoles in the Boltzmann hierarchy. Alternatively, one may treat all the species through a Boltzmann hierarchy. This is the second, complementary, approach to the problem of initial conditions.

Proceeding along the improved fluid approach the covariant conservation equations for photons and baryons imply, respectively,

$$
\begin{aligned}
& \theta_{\mathrm{b}}^{\prime}+\mathcal{H} \theta_{\mathrm{b}}=k^{2} \phi+\frac{\mathcal{F}_{\mathrm{B}}(k)}{4 \pi a^{4} \rho_{\mathrm{b}}}+\frac{4}{3} \frac{\Omega_{\gamma}}{\Omega_{\mathrm{b}}} a n_{\mathrm{e}} x_{\mathrm{e}} \sigma_{\mathrm{T}}\left(\theta_{\gamma}-\theta_{\mathrm{b}}\right)=0 \\
& \delta_{\mathrm{b}}^{\prime}=3 \psi^{\prime}-\theta_{\mathrm{b}} .
\end{aligned}
$$

and

$$
\begin{aligned}
& \delta_{\gamma}^{\prime}=-\frac{4}{3} \theta_{\gamma}+4 \psi^{\prime} \\
& \theta_{\gamma}^{\prime}=\frac{k^{2}}{4} \delta_{\gamma}+k^{2} \phi+a n_{\mathrm{e}} \sigma_{\mathrm{T}}\left(\theta_{\mathrm{b}}-\theta_{\gamma}\right)
\end{aligned}
$$

In Eqs. (5.46) and (5.49) Thompson scattering term has been added. At early times, baryons and photons are synchronized so well that, to lowest order in the tight coupling approximation, $\sigma_{\mathrm{T}} \rightarrow \infty$ and $\theta_{\gamma} \simeq \theta_{\mathrm{b}}$. 
The evolution of the CDM particles follows from the fluctuations of the covariant conservation equation whose first-order fluctuation leads, in Fourier space, to

$$
\begin{aligned}
& \theta_{\mathrm{c}}^{\prime}+\mathcal{H} \theta_{c}=k^{2} \phi, \\
& \delta_{\mathrm{c}}^{\prime}=3 \psi^{\prime}-\theta_{\mathrm{c}} .
\end{aligned}
$$

To describe neutrinos during and after horizon crossing requires a Boltzmann hierarchy for $\delta_{\nu}, \theta_{\nu}$ and for the higher multipole moments, i.e. $\ell \geq 2$, of the neutrino phase-space density $\mathcal{F}_{\nu \ell}$. With this caveat in mind, after neutrino decoupling, at temperatures of about $1 \mathrm{MeV}$, massless neutrinos obey, in Fourier space, the following set of equations

$$
\begin{aligned}
\delta_{\nu}^{\prime} & =-\frac{4}{3} \theta_{\nu}+4 \psi^{\prime}, \\
\theta_{\nu}^{\prime} & =\frac{k^{2}}{4} \delta_{\nu}-k^{2} \sigma_{\nu}+k^{2} \phi, \\
\sigma_{\nu}^{\prime} & =\frac{4}{15} \theta_{\nu}-\frac{3}{10} k \mathcal{F}_{\nu 3},
\end{aligned}
$$

where $\sigma_{\nu}=\mathcal{F}_{\nu 2} / 2$ is the quadrupole moment of the (perturbed) neutrino phase-space distribution and, as introduced above, $\mathcal{F}_{\nu \ell}$ is the $\ell$-th multipole.

The closed system formed by the perturbed Einstein equations and by the fluid equations may be solved. For illustrative purposes, consider the magnetized adiabatic mode in the force-free case. The solution can be obtained as an expansion in $k \tau$ which is small, i.e. $k \tau<1$ when the relevant modes are outside the horizon. In the presence of a magnetic field the density contrasts turn out to be

$$
\begin{aligned}
& \delta_{\mathrm{b}, \mathrm{c}}=\bar{\delta}_{\mathrm{b}, \mathrm{c}}-\frac{3}{4} \Omega_{\mathrm{B}}-\left[\frac{69-61 R-8 R_{\nu}^{2}}{60\left(25+2 R_{\nu}\right)}\right] \Omega_{\mathrm{B}} k^{2} \tau^{2}, \\
& \delta_{\gamma}=\bar{\delta}_{\gamma}-\Omega_{\mathrm{B}}+\left[\frac{237+152 R_{\nu}+16 R_{\nu}^{2}}{90\left(25+2 R_{\nu}\right)}\right] \Omega_{\mathrm{B}} k^{2} \tau^{2}, \\
& \delta_{\nu}=\bar{\delta}_{\nu}-\Omega_{\mathrm{B}}-\left[\frac{375-207 R_{\nu}-152 R_{\nu}^{2}-16 R_{\nu}^{3}}{90 R_{\nu}\left(25+2 R_{\nu}\right)}\right] \Omega_{\mathrm{B}} k^{2} \tau^{2},
\end{aligned}
$$

where the barred quantities denote the adiabatic mode in the absence of magnetic field. In particular, for the density contrasts in the absence of inhomogeneous magnetic field we have

$$
\begin{aligned}
& \bar{\delta}_{\mathrm{b}}=\bar{\delta}_{\mathrm{c}}=-\frac{3}{2} \phi_{0}-\frac{\left(525+188 R_{\nu}+16 R_{\nu}^{2}\right)}{60\left(25+2 R_{\nu}\right)} \phi_{0} k^{2} \tau^{2}, \\
& \bar{\delta}_{\gamma}=\bar{\delta}_{\nu}=-2 \phi_{0}-\frac{\left(525+188 R_{\nu}+16 R_{\nu}^{2}\right)}{45\left(25+2 R_{\nu}\right)} \phi_{0} k^{2} \tau^{2},
\end{aligned}
$$

where $\phi_{0}$ is the constant value of $\phi$ to lowest order in $k \tau$. While in the absence of magnetic field the adiabaticity condition of Eq. (5.34) is verified both to lowest and to higher order in 
$k \tau$, in the presence of magnetic field the adiabaticity condition is violated to next-to-leading order.

With similar notations we can also report the evolution for the metric fluctuations

$$
\begin{aligned}
& \phi=\bar{\phi}-\left[\frac{6-8 R_{\nu}+2 R_{\nu}^{2}}{45\left(25+2 R_{\nu}\right)} \Omega_{\mathrm{B}}\right] k^{2} \tau^{2}, \\
& \psi=\bar{\psi}-\left[\frac{69-61 R_{\nu}-8 R_{\nu}^{2}}{180\left(25+2 R_{\nu}\right)} \Omega_{\mathrm{B}}\right] k^{2} \tau^{2},
\end{aligned}
$$

where, as before, $\bar{\phi}$ and $\bar{\psi}$ denote the standard adiabatic solution, i.e.

$$
\begin{aligned}
& \bar{\phi}=\phi_{0}-\frac{\left(75+14 R_{\nu}-8 R_{\nu}^{2}\right)}{90\left(25+2 R_{\nu}\right)} \phi_{0} k^{2} \tau^{2}, \\
& \bar{\psi}=\psi_{0}-\frac{\left(75+79 R_{\nu}+8 R_{\nu}^{2}\right)}{90\left(25+2 R_{\nu}\right)} \phi_{0} k^{2} \tau^{2} .
\end{aligned}
$$

As in the standard adiabatic case, $\psi_{0}$ is determined in terms of $\phi_{0}$ by the neutrino anisotropic stress. In the magnetized case the relation is modified as

$$
\psi_{0}=\phi_{0}\left(1+\frac{2}{5} R_{\nu}\right)-\frac{\Omega_{\mathrm{B}}}{5}\left(R_{\nu}-1\right) .
$$

The solution for the velocity fields is finally

$$
\begin{aligned}
& \theta_{\gamma}=\bar{\theta}_{\gamma}-\left[\frac{\Omega_{\mathrm{B}}}{4} k^{2} \tau+\frac{\left(7+8 R_{\nu}\right)}{40(25+2 R)} \Omega_{\mathrm{B}} k^{4} \tau^{3}\right], \\
& \theta_{\nu}=\bar{\theta}_{\nu}-\frac{\Omega_{\mathrm{B}}}{4} \frac{R_{\gamma}}{R_{\nu}} k^{2} \tau-\left[\frac{45-29 R_{\nu}-16 R_{\nu}^{2}}{72 R_{\nu}\left(25+2 R_{\nu}\right)} \Omega_{\mathrm{B}}\right] k^{4} \tau^{3}, \\
& \theta_{\mathrm{b}}=\theta_{\mathrm{c}}=\bar{\theta}_{\mathrm{b}}-\left[\frac{6-8 R_{\nu}+2 R_{\nu}^{2}}{180\left(25+2 R_{\nu}\right)} \Omega_{\mathrm{B}}\right] k^{4} \tau^{3},
\end{aligned}
$$

where, following the same notations used before, the standard adiabatic solution is

$$
\begin{aligned}
& \bar{\theta}_{\nu}=\frac{\phi_{0}}{2} k^{2} \tau-\frac{\left(65+16 R_{\nu}\right)}{36\left(25+2 R_{\nu}\right)} \phi_{0} k^{4} \tau^{3}, \\
& \bar{\theta}_{\mathrm{b}}=\frac{\phi_{0}}{2} k^{2} \tau-\frac{\left(75+14 R_{\nu}-8 R_{\nu}^{2}\right)}{360\left(25+2 R_{\nu}\right)} \phi_{0} k^{4} \tau^{3}, \\
& \bar{\theta}_{\mathrm{c}}=\frac{\phi_{0}}{2} k^{2} \tau-\frac{\left(75+14 R_{\nu}-8 R_{\nu}^{2}\right)}{360\left(25+2 R_{\nu}\right)} \phi_{0} k^{4} \tau^{3}, \\
& \bar{\theta}_{\gamma}=\frac{\phi_{0}}{2} k^{2} \tau-\frac{\left(25+8 R_{\nu}\right)}{20\left(25+2 R_{\nu}\right)} \phi_{0} k^{2} \tau^{2},
\end{aligned}
$$

Notice that while to leading order all the peculiar velocities of the plasma coincide, to next-to-leading order they are different: this occurs already in the standard adiabatic case.

Since the adiabaticity condition is only realized to lowest order in $k \tau$ it is legitimate to name the solution presented in Eqs. (5.55)-(5.67) quasi-adiabatic. The corrections to 
adiabaticity are of order $\Omega_{\mathrm{B}} k^{2} \tau^{2}$, so they are suppressed outside the horizon and also, by virtue of Eq. (5.42), by $\Omega_{\mathrm{B}}$. Notice that three regimes emerge naturally. The quasiadiabatic regime where $\Omega_{\mathrm{B}}(k) \leq \phi_{0}(k)$, the isocurvature regime $\Omega_{\mathrm{B}}(k)>\phi_{0}(k)$ and the fully adiabatic regime, i.e. $\Omega_{\mathrm{B}}(k) \ll \phi_{0}(k)$.

It is useful to recall that the presence of fully inhomogeneous magnetic fields changes the qualitative features of the tight coupling expansion 113. As it is well known, the idea of the tight-coupling approximation is to tailor a systematic expansion in powers of the inverse optical depth, i.e. $1 / \epsilon^{\prime}$ in the notations employed Eq. (3.40). Instead of being interested in the brightness perturbations related to $\mathrm{Q}$ and $\mathrm{U}$ we shall be interested in the brightness perturbation related to I, i.e. the intensity of the scattered radiation. To derive the evolution equations in the tight-coupling approximation for a magnetoactive plasma we recall that the equation for $\Delta_{\mathrm{I}}$ can be written as

$$
\Delta_{\mathrm{I}}^{\prime}+i k \mu \Delta_{\mathrm{I}}=\left(\psi^{\prime}-i k \mu \phi\right)+\tau^{\prime}\left[-\Delta_{\mathrm{I}}+\Delta_{\mathrm{I} 0}+\mu v_{\mathrm{b}}-\frac{1}{2} P_{2}(\mu) S_{\mathrm{Q}}\right],
$$

where we defined $v_{\mathrm{b}}=\theta_{\mathrm{b}} /(i k)$ and where $S_{\mathrm{Q}}$ is the same quantity already introduced in Eq. (3.51). The equation for $v_{\mathrm{b}}$ can be readily obtained from Eq. (5.46) and it is given by

$$
v_{\mathrm{b}}^{\prime}+\mathcal{H} v_{\mathrm{b}}=-i k \phi-\frac{\tau^{\prime}}{\alpha}\left[3 i \Delta_{\mathrm{I} 1}+v_{\mathrm{b}}\right]-i \mathcal{W}_{\mathrm{B}}(k),
$$

where

$$
\begin{aligned}
& \mathcal{W}_{\mathrm{B}}(k)=\frac{\mathcal{F}_{\mathrm{B}}}{4 \pi k \rho_{\mathrm{b}} a^{4}}, \\
& \alpha=\frac{3}{4} \frac{\rho_{\mathrm{b}}}{\rho_{\gamma}} .
\end{aligned}
$$

It should be noticed that even if the magnetic field does not appear directly in Eq. (5.72), it does appear in Eq. (5.73). This implies that the equation for $\Delta_{\mathrm{I}}$ (already to zeroth order in the tight-coupling expansion) inherits an extra source term that depend on the divergence of the Lorentz force. This statement can be verified by expanding, consistently, the evolution equations for the brightness perturbations in powers of $1 /\left|\epsilon^{\prime}\right|$ [113]. For instance, to zeroth order in the tight-coupling expansion the (decoupled) evolution equation for the monopole can be writtten as

$$
\bar{\Delta}_{\mathrm{I} 0}^{\prime \prime}+\frac{\alpha^{\prime}}{\alpha+1} \bar{\Delta}_{\mathrm{I} 0}^{\prime}+\frac{k^{2}}{3(\alpha+1)} \bar{\Delta}_{\mathrm{I} 0}=\left[\psi^{\prime \prime}+\frac{\alpha^{\prime}}{\alpha+1} \psi^{\prime}-\frac{k^{2}}{3} \phi\right]-\frac{\alpha}{\alpha+1} \frac{\mathcal{F}_{\mathrm{B}}}{12 \pi \rho_{\mathrm{b}} a^{4}} .
$$

In the limit $\mathcal{F}_{\mathrm{B}} \rightarrow 0$ the usual decoupled equation for the evolution of the monopole is clearly recovered. 


\subsection{Constraints on fully inhomogeneous fields}

In the following we will refer exclusively to the fully inhomogeneous case since the uniform case has been already discussed in Section 3. In [115] the effects of a fully inhomogeneous magnetic field has been considered semi-analytically. The analysis of [115] was motivated by the results of where, in a specific model, quite sizable large-scale magnetic fields could be onbtained. In particular, the energy spectrum could be, in that case, quasi-flat, i.e. in the notations of Section $4, m \simeq-2.9$. It was then noted that a rough limit on the magnetic field intensity could be obtained, i.e. $B_{L} \leq 10^{-9}$ where we follow the notations of Section 4 and take as comoving scale of regularization the Mpc.

A semi-analytical analysis of the tensor and vector modes performed in [72]. The authors discussed correctly two opposite regime. In the case $m<-3 / 2$ the two-point function of the Lorentz force is dominated by the large-scale features of the magnetic field. On the contrary, if $m>-3 / 2$ the two-point function of the Lorentz force is dominated by the high $k$-modes and hence by diffusion. In fact, the power-spectrum of the Lorentz force term can be estimated as

$$
P(k) \simeq \frac{1}{\pi(2 m+3)}\left[\frac{(2 \pi)^{m+3} B_{L}^{2}}{\Gamma\left(\frac{n+3}{2}\right) \rho_{\gamma}}\right]^{2}\left[\left(\frac{k_{D}}{k_{L}}\right)^{2 m+3}\left(\frac{k}{k_{L}}\right)^{3}+\frac{m}{m+3}\left(\frac{k}{k_{L}}\right)^{2 n+3}\right],
$$

where $k_{D}$ is the diffusion scale. The diffusion scale can be estimated, for instance, from the considerations of Ref. [16] where it was argued that the diffusion length-scale is basically determined by the Alfvén velocity times the Silk length-scale. From Eq. (5.77) we can see that the second term dominates for $-3<m<-3 / 2$ while the first term dominates for $m>-3 / 2$. For $m=-3 / 2$ both terms may be of the same order. Notice that, in Eq. (5.77), $k_{L}=2 \pi / L$ and $L$ is the regularization length-scale introduced at the end of the previous Section (see Eq. (4.22)).

In 72 the $\mathrm{TT}, \mathrm{EE}, \mathrm{TE}$ and $\mathrm{BB}$ correlations ${ }^{16}$ for vector and tensor modes have been estimated semi-analytically. Using $10 \%$ estimates the authors of Ref. 72 obtain for $m \sim-3$ (e.g. $m \sim-2.9$ ) a limit $B_{L} \leq 7 \times 10^{-9}$ G. This type of limit becomes more stringent as $m$ increases: for $m=0$ we have $B_{\mathrm{L}} \leq 10^{-10} \mathrm{G}$, while for $m \simeq 2$ we would have $B_{\mathrm{L}} \leq 10^{-13} \mathrm{G}$.

Recently in [11] the calculation of the vector and tensor modes has been pushed to $\ell \sim 2000$. It is clear from the reported results [111] that for a magnetic field with $B_{L} \sim$ $10^{-9} \mathrm{G}$ and $m \sim-2.9$ the TT correlations induced by the vector modes become of the same order to the TT power spectrum (in the absence of magnetic field) for $\ell \geq 2000$. Expected numerical disagreements with the semi-analytical estimates of [72] have been also found for $\ell \geq 100$. In Fig. [5 the results obtained in the case of vectors for a typical magnetic field

\footnotetext{
${ }^{16}$ See the discussion in Section 3 on the cross power spectra whose general definition is given in Eq. (3.66).
} 


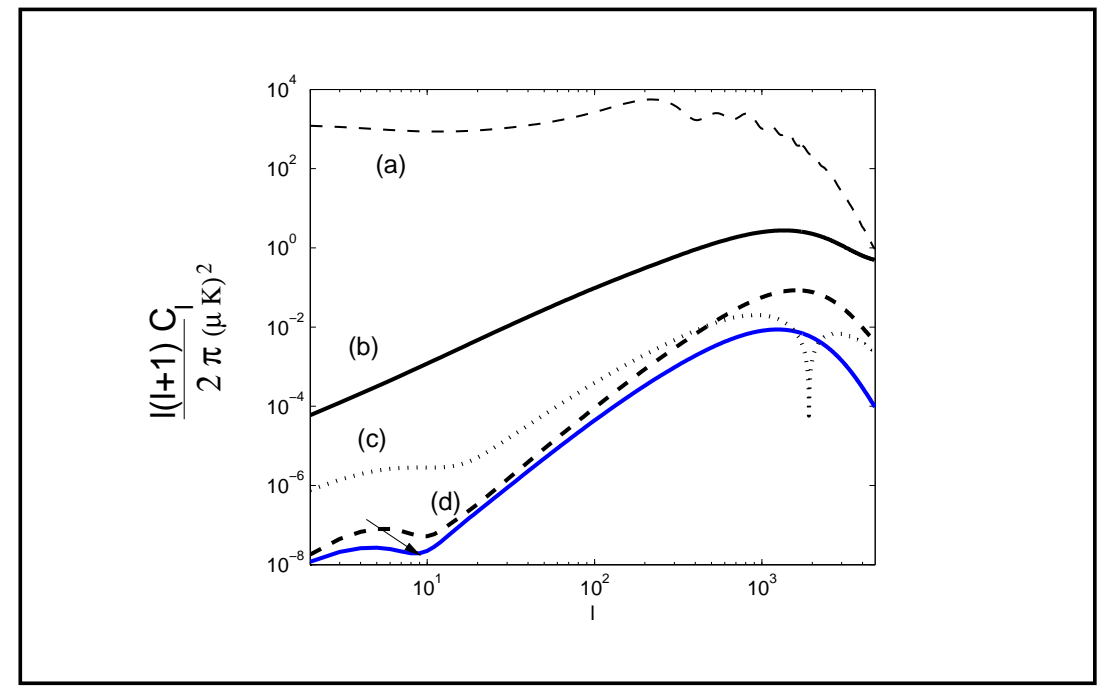

Figure 5: CMB power spectra (vector modes) for $B_{L} \sim 3 \times 10^{-9} \mathrm{G}$. Adapted from Ref. [110]. TT correlations in the absence of magnetic field (curve (a)); TT correlations in the presence of magnetic fields (curve (b)); absolute value of TE correlation (curve (c)); BB correlations (curve (d)); EE correlation (indicated by the arrow). These correlations have been defined in Section 3, see, in particular, the discussion from Eq. (3.61) to Eq. (3.71); the notation EE, BB, TE, and so on, refers to the cross power spectra defined in Eq. (3.66) (see also the text). 
$B_{L} \sim 3 \times 10^{-9} \mathrm{G}$ are reported (see [110]).

Scalar modes have been less studied than the vector and tensor modes. The expectation is that scalar modes do not give stronger constraints than vectors or tensors. It would be, however, desirable to bring the analysis of the scalar problem to the same standard of ordinary CMB calculations where the adiabatic mode and the different isocurvature modes are constrained to a given confidence level. For instance the results reported by [114 are difficult to intepret since bounds on the magnetic field intensity are claimed but the initial conditions for the analysis are not clearly specified.

\subsection{Other effects}

On top on the effects of large-scale magnetic fields on anisotropies there are effects directly connected with CMB spectrum like the possible distorsions. These have been studied in Ref. [117] (see also [118]). The possible distortions of the Planckian spectrum of CMB are usually discussed in terms of a chemical potential which is bounded, by experimental data, to me $|\mu|<9 \times 10^{-5}$. Magnetic field dissipation at high red-shift implies the presence of a chemical potential. Hence bounds on the distorsion of the Planckian spectrum of the CMB can be turned into bounds on the magnetic field strength at various scales. In particular [117] the obtained bound are such that $B<3 \times 10^{-8} \mathrm{G}$ for comoving coherence lengths between $0.4 \mathrm{kpc}$ and $500 \mathrm{kpc}$.

As in the case of synchrotron emission also Faraday rotation measurements can be used as a diagnostic for foreground contamination. The idea would be, in this context to look for cross-correlations in the Faraday rotation measure of extra-galactic sources and the measured microwave signal at the same angular position. A recent analysis has been recently reported [119]. (see also [120, 121, 122]).

\section{Concluding remarks}

Simple logic dictates that if the origin of the large-scale magnetic fields is primordial (as opposed to astrophysical) it is plausible to expect the presence of magnetic fields in the primeval plasma also before the decoupling of radiation from matter. As it was argued in the present topical review, CMB anisotropies are germane to several aspect of large-scale magnetization. CMB physics may be the tool that will finally enable us either to confirm or to rule out the primordial nature of galactic and clusters magnetic fields seeds. In the next

five to ten years the forthcoming $\mathrm{CMB}$ precision polarization experiments will be sensitive in, various frequency channels between above100 GHz and, roughly below $900 \mathrm{GHz}$. The 
observations will be conducted both via satellites (like the Planck satellite) and via ground based detectors (like in the case of the QUIET arrays). In a complementary view, the SKA telescope will provide full-sky surveys of Faraday rotation that may even get close to 20 $\mathrm{GHz}$.

In an optimistic perspective the forthcoming experimental data together with the steady progress in the understanding of the dynamo theory will hopefully explain the rationale for the ubiquitous nature of large-scale magnetization. In a pessimistic perspective, the primordial nature of magnetic seeds will neither be confirmed nor ruled out. The plausibility of (highly speculative) theoretical scenarios for the origin of primordial magnetic fields cannot be decided only using the fertile imagination of theoretical physicists. It would be wiser, at the same time, to adopt a model-independent approach by sharpening those theoretical tools that may allow, in the near future, a direct observational test of the effects of large-scale magnetic fields on CMB anisotropies. 


\section{References}

[1] Ya. Zeldovich, Sov. Phys. JETP 21, 656 (1965).

[2] Ya. Zeldovich and I. Novikov, The Structure and Evolution of the Universe, (Chicago University Press, Chicago, 1971), Vol.2.

[3] I. M. Khalatnikov, Sov. Phys. JETP 21, 172 ( 1965).

[4] E. M. Lifshitz and I. M. Khalatnikov, Sov. Phys. JETP 12, 108 (1960).

[5] E. M. Lifshitz and I. M. Khalatnikov, Sov. Phys. JETP 12, 558 (1961).

[6] V. A. Belinskii and I. M. Khalatnikov, Sov. Phys. JETP 30, 1174 (1970).

[7] M.P. Ryan, and L.C. Shepley, Homogeneous Relativistic Cosmologies, ( Princeton University Press, Princeton 1975).

[8] J. D. Barrow, Phys. Rev. D 55, 7451 (1997).

[9] J. D. Barrow, P. G. Ferreira and J. Silk, Phys. Rev. Lett. 78, 3610 (1997).

[10] M. Giovannini, Phys. Rev. D 59, 123518 (1999).

[11] J. D. Barrow and R. Maartens, Phys. Rev. D 59, 043502 (1999).

[12] M. Giovannini, Phys. Rev. D 62, 067301 (2000).

[13] K. A. Bronnikov, E. N. Chudaeva and G. N. Shikin, Class. Quant. Grav. 21, 3389 (2004).

[14] G. Chen et al., Astrophys. J. 611, 655 (2004).

[15] R. Durrer, T. Kahniashvili and A. Yates, Phys. Rev. D 58, 123004 (1998).

[16] K. Subramanian and J. D. Barrow, Phys. Rev. D 58, 083502 (1998).

[17] K. Subramanian and J. D. Barrow, Phys. Rev. Lett. 81, 3575 (1998).

[18] T. R. Seshadri and K. Subramanian, Phys. Rev. D 72, 023004 (2005).

[19] J.Adams, U.Danielsson, D.Grasso, and H. Rubinstein, Phys. Lett. B 388, 253 (1996).

[20] D. Grasso and H. R. Rubinstein, Phys. Rept. 348, 163 (2001).

[21] A. Vlasov, Zh. Éksp. Teor. Fiz. 8, 291 (1938); J. Phys. 9 (Moscow), 25 (1945).

[22] L. D. Landau, J. Phys. (Moscow) 10, 25 (1945). 
[23] E. M. Lifshitz and L. P. Pitaevskii, Physical Kinetics, (Pergamon Press, Oxford, England, 1980).

[24] D. Biskamp, Non-linear Magnetohydrodynamics (Cambridge University Press, Cambridge, 1994).

[25] T. J. M Boyd and J. J. Sanderson, The physics of plasmas, (Cambridge University Press, Cambridge, UK, 2003).

[26] M. Giovannini, Phys. Rev. D 71, 021301 (2005).

[27] J. Skilling, Phys. of Fluids 14, 2523 (1971).

[28] S. M. Carroll, G. B. Field and R. Jackiw, Phys. Rev. D 41, 1231 (1990).

[29] S. M. Carroll, Phys. Rev. Lett. 81, 3067 (1998).

[30] M. Giovannini, Phys. Rev. D 61, 063004 (2000).

[31] M. Giovannini, Phys. Rev. D 61, 063502 (2000).

[32] B. Feng, H. Li, M. z. Li and X. m. Zhang, Phys. Lett. B 620, 27 (2005).

[33] . K. Sethi and K. Subramanian, Mon. Not. Roy. Astron. Soc. 356, 778 (2005).

[34] K. R. S. Balaji, R. H. Brandenberger and D. A. Easson, JCAP 0312, 008 (2003).

[35] A. Lue, L. M. Wang and M. Kamionkowski, Phys. Rev. Lett. 83, 1506 (1999).

[36] N. F. Lepora, arXiv gr-qc/9812077.

[37] A. Kosowsky and A. Loeb, Astrophys. J 461, 1 (1996).

[38] M. Giovannini, Phys. Rev. D 56, 3198 (1997).

[39] D. Harari, J. Hayward and M. Zaldarriaga, Phys. Rev. D 55, 1841 (1997).

[40] M. Giovannini, Int. J. Mod. Phys. D 14, 363 (2005).

[41] J. J. Sakurai, Modern Quantum Mechanics, (Addison-Wesley, New York, 1985).

[42] C.-P. Ma and E. Bertschinger, Astrophys. J. 455, 7 (1995).

[43] W. Hu and N. Sugiyama, Astrophys. J. 444, 489 (1995).

[44] H. V. Peris et al., Astrophys. J. Suppl. 148, 161 (2003).

[45] A. Kogut et al., Astrophys. J. Suppl. 148, 213 (2003). 
[46] C. Scoccola, D. Harari, and S. Mollerach, Phys. Rev. D 70, 063003 (2004).

[47] L. Campanelli, A. D. Dolgov, M. Giannotti and F. L. Villante, Astrophys. J. 616, 1 (2004).

[48] A. D. Dolgov, Lectures given at the International School on Astrophysics 'Daniel Chalonge' ( 8th Paris Cosmology Colloquium 2004 and Topical Meeting WMAP and the Early Universe, Paris, France, 9-10 Dec 2004), arXiv astro-ph/0503447.

[49] A. Kosowsky, T. Kahniashvili, G. Lavrelashvili and B. Ratra, Phys. Rev. D 71, 043006 (2005).

[50] see, for instance, http://www.rssd.esa.int/index.php?project=PLANCK.

[51] see, for instance, http://oberon.roma1.infn.it/boomerang/b2k/

[52] S. Masi et al., arXiv astro-ph/0507509.

[53] see, for instance, http://cfcpwork.uchicago.edu/kicp-projects/quiet/.

[54] see, for instance, http://www.skads-eu.org/

[55] F. Govoni and L. Feretti, Int. J. Mod. Phys. D 13, 1549 (2004).

[56] B. M. Gaensler, R. Beck and L. Feretti, New Astron. Rev. 48, 1003 (2004).

[57] N. A. Krall and A. W. Trivelpiece, Principles of Plasma Physics, (San Francisco Press, San Francisco 1986).

[58] D. T. Son, Phys. Rev. D 59, 063008 (1999).

[59] A. Brandenburg, K. Enqvist and P. Olesen Phys. Rev. D 54, 1291 (1996).

[60] A. Brandenburg, K. Enqvist and P. Olesen Phys. Lett. B 392 , 395 (1997).

[61] P. Olesen, Phys. Lett. B 398, 321 (1997).

[62] K. Enqvist, Int. J. Mod. Phys. D 7, 331 (1998).

[63] M. Giovannini, Int. J. Mod. Phys. D 13, 391 (2004).

[64] L. M. Widrow, Rev. Mod. Phys. 74, 775 (2003).

[65] L. Pogosian, T. Vachaspati, and S. Winitzki Phys.Rev.D 65, 083502 (2002).

[66] M. Giovannini and M. E. Shaposhnikov, Phys. Rev. Lett. 80, 22 (1998).

[67] M. Giovannini and M. E. Shaposhnikov, Phys. Rev. D 57, 2186 (1998). 
[68] T. Kahniashvili and B. Ratra, Phys. Rev. D 71, 103006 (2005).

[69] M. Abramowitz and I. A. Stegun, Handbook of Mathematical Functions (Dover, New York, 1972).

[70] I. S. Gradshteyn and I. M. Ryzhik, Tables of Integrals, Series and Products (fifth edition), (Academic Press, New York, 1994).

[71] M. Giovannini and M. E. Shaposhnikov, Phys. Rev. D 62, 103512 (2000).

[72] A. Mack, T. Kahniashvili and A. Kosowsky, Phys. Rev. D 65, 123004 (2002).

[73] K. Kajantie, et al. Phys. Rev. Lett. 77, 2887 (1996).

[74] T. Kibble and A. Vilenkin, Phys. Rev. D 52, 679 (1995).

[75] J. Ahonen and K. Enqvist, Phys. Rev. D 57, 664 (1997).

[76] G. Baym, D. Bodeker and L. McLerran, Phys. Rev. D 53, 662 (1996).

[77] J. Quashnock, A. Loeb and D. Spergel, Astrophys. J. Lett. 344, L49 (1989).

[78] B. Cheng and A. Olinto, Phys. Rev. D 50, 2421 (1994).

[79] G. Sigl, A. Olinto, and K. Jedamzik, Phys. Rev. D55, 4852 (1997).

[80] A. D. Dolgov, hep-ph/0110293.

[81] M. Giovannini, Phys. Rev. D 62, 123505 (2000).

[82] M. Giovannini, Phys. Rev. D 64, 061301 (2001).

[83] K. Bamba and J. Yokoyama, Phys. Rev. D 70, 083508 (2004).

[84] O. Bertolami and R. Monteiro, Phys. Rev. D 71, 123525 (2005).

[85] K. E. Kunze, Phys. Lett. B 623, 1 (2005).

[86] M. Gasperini, M. Giovannini and G. Veneziano, Phys. Rev. Lett. 75, 3796 (1995).

[87] D. Boyanovsky, M. Simionato and H. J. de Vega, Phys. Rev. D 67, 023502 (2003).

[88] D. Boyanovsky, H. J. de Vega and M. Simionato, Phys. Rev. D 67, 123505 (2003).

[89] D. Boyanovsky and H. J. de Vega, arXiv astro-ph/0502212.

[90] K. Enqvist, A. Jokinen and A. Mazumdar, JCAP 0411, 001 (2004). 
[91] A. Lazarian, Astron. Astrophys. 264, 326 (1992).

[92] M. J. Rees, Lect. Notes Phys. 664, 1 (2005).

[93] A. Lazarian, E. Vishniac and J. Cho, Astrophys. J. 603, 180 (2004); Lect. Notes Phys. 614, 376 (2003).

[94] A. Brandenburg and K. Subramanian, Phys. Rept. 417, 1 (2005).

[95] R. Kulsrud, Annu. Rev. Astron. Astrophys. 37, 37 (1999).

[96] R. Kulsrud and S. Anderson, Astrophys. J. 396, 606 (1992).

[97] I. Brown and R. Crittenden, Phys. Rev. D 72, 063002 (2005).

[98] J. M. Bardeen, Phys. Rev. D 22, 1882 (1980).

[99] C. G. Tsagas and J. D. Barrow, Class. Quant. Grav. 14, 2539 (1997).

[100] C. Tsagas and R. Maartens, Phys. Rev. D 61, 083519 (2000).

[101] C. G. Tsagas, Class. Quant. Grav. 22, 393 (2005)

[102] M. Giovannini, Phys. Rev. D 58, 124027 (1998).

[103] T. Kahniashvili, G. Gogoberidze and B. Ratra, arXiv astro-ph/0505628

[104] C. G. Tsagas, Class. Quant. Grav. 19, 3709 (2002).

[105] T. J. Battefeld and R. Brandenberger, Phys. Rev. D 70, 121302 (2004).

[106] M. Giovannini, Phys. Rev. D 70, 103509 (2004).

[107] M. Giovannini, Class. Quant. Grav. 22, 363 (2005).

[108] K . Subramanian and J. D. Barrow, Mon. Not. Roy. Astron. Soc. 335, L57 (2002).

[109] K. Subramanian, T. R. Seshadri and J. D. Barrow, Mon. Not. Roy. Astron. Soc. 344, L31 (2003).

[110] A. Lewis, Phys. Rev. D 70, 043011 (2004).

[111] A. Lewis, Phys. Rev. D 70, 043518 (2004).

[112] M. Giovannini, Phys. Lett. B 622, 349 (2005).

[113] M. Giovannini, Phys. Rev. D 70, 123507 (2004). 
[114] S. Koh and C. H. Lee, Phys. Rev. D 62,083509 (2000).

[115] M. Gasperini, M. Giovannini and G. Veneziano, Phys. Rev. D 52, 6651 (1995).

[116] C. A. Clarkson, A. A. Coley, R. Maartens and C. G. Tsagas, Class. Quant. Grav. 20, 1519 (2003).

[117] K. Jedamzik, V. Katalinic and A. V. Olinto, Phys. Rev. Lett. 85, 700 (2000).

[118] A. Zizzo and C. Burigana, arXiv astro-ph/0505259.

[119] P. Dineen and P. Coles, Mon. Not. Roy. Astron. Soc. 347, 52 (2004).

[120] G. Bernardi, E. Carretti, R. Fabbri, C. Sbarra and S. Cortiglioni, arXiv astro-ph/0508278.

[121] P. D. Naselsky, A. G. Doroshkevich and O. V. Verkhodanov, Astrophys. J. 599, L53 (2003).

[122] P. D. Naselsky, L-Y. Chiang, P. Olesen, O. Verkhodanov, Astrophys. J. 615, 45 (2004). 